\title{
Modeling peer effect modification by network strength: The diffusion of implantable cardioverter defibrillators in the US hospital network
}

\author{
A. James O'Malley ${ }^{1,2}$ (i) $\mid$ Erika L. Moen ${ }^{1} \mid$ Julie P. W. Bynum $^{3}$ \\ Andrea M. Austin ${ }^{2}$ | Jonathan S. Skinner1,4
}

${ }^{1}$ Department of Biomedical Data Science, Geisel School of Medicine at Dartmouth, Hanover, New Hampshire

${ }^{2}$ The Dartmouth Institute of Health Policy and Clinical Practice, Geisel School of Medicine at Dartmouth, Hanover, New Hampshire

${ }^{3}$ Department of Medicine, University of Michigan, Ann Arbor, Michigan

${ }^{4}$ Department of Economics, Dartmouth College, Hanover, New Hampshire

\section{Correspondence}

A. James O'Malley, The Dartmouth Institute of Health Policy and Clinical Practice, Geisel School of Medicine at Dartmouth, 1 Medical Center Drive, Geisel School of Medicine at Dartmouth, Lebanon, NH 03756, USA.

Email: James.OMalley@Dartmouth.edu

\section{Funding information}

National Institutes of Health (NIH), United States of America, P01 AG019783, U01 AG046830
We develop methodology that allows peer effects (also referred to as social influence and contagion) to be modified by the structural importance of the focal actor's position in the network. The methodology is first developed for a single peer effect and then extended to simultaneously model multiple peer-effects and their modifications by the structural importance of the focal actor. This work is motivated by the diffusion of implantable cardioverter defibrillators (ICDs) in patients with congestive heart failure across a cardiovascular disease patient-sharing network of United States hospitals. We apply the general methodology to estimate peer effects for the adoption of capability to implant ICDs, the number of ICD implants performed by hospitals that are capable, and the number of patients referred to other hospitals by noncapable hospitals. Applying our novel methodology to study ICD diffusion across hospitals, we find evidence that exposure to ICD-capable peer hospitals is strongly associated with the chance a hospital becomes ICD-capable and that the direction and magnitude of the association is extensively modified by the strength of that hospital's position in the network, even after controlling for effects of geography. Therefore, interhospital networks, rather than geography per se, may explain key patterns of regional variations in healthcare utilization.

\section{K E Y W O R D S}

hierarchical model, implantable cardioverter defibrillator, longitudinal model, nationwide hospital network, peer effect, social network analysis

\section{1 | INTRODUCTION}

Over the past 40 years, dramatic geographic differences across the United States (US) in healthcare utilization and outcomes have been widely documented. ${ }^{1-6}$ Furthermore, rates of diffusion for new medical technologies vary widely across countries and areas, but little is understood about why some health organizations are more likely to adopt while others lag behind. Social networks whose nodes are entities (eg, hospitals) who may adopt the technology and whose edges reflect relationships between the nodes have been considered integral to the diffusion of medical technologies for many years. ${ }^{7}$ However, nationwide networks reflecting the potential for information exchange, policy sharing and other forms 
of effective healthcare collaboration have not been examined in relation to adoption and level of utilization of medical technologies across the US healthcare system.

We are generally interested in whether peer effects-the extent to which adoption of similar practices or behaviors is due to the transmission and receipt of influence through a network-exist and, if so, whether they depend on the position of the focal actor in the network. This interest stems from the hypothesis that the actors with the greatest structural importance in the network might be influenced differently by their peers than an actor in a structurally weak (eg, peripheral) position. For example, in a network of hospitals, the structural importance of a hospital may reflect its potential to attract patients via hidden alliances with other hospitals. In the case of a hospital adopting an expensive technology requiring highly specialized operators such as implantable cardiac defibrillators (ICDs), a theoretical basis exists for peer effect modification by an actor's network strength. A hospital with high structural importance may be positively influenced toward adoption by a high rate of adoption of their peer hospitals (those they have edges with in the network), whereas a hospital in a weak position may be negatively influenced. We further hypothesize that the likelihood of continuing as ICD capable will also depend on peer effects that are modified by the focal hospital's network strength. In this motivating application, structural importance will be measured using the sum of the values of a hospital's edges with other hospitals in the network, a quantity commonly known as network "strength." However, the methodology applies to any measure of structural importance such as any of the centrality measures or simply using network degree (the number of distinct hospitals it shares patients with - the unweighted counterpart of network strength).

Models of peer effects are typically similar in form to conditional autoregressive ${ }^{8}$ and simultaneous autoregressive ${ }^{8-11}$ models used in spatial statistics and econometrics. In these models, the focus is on determining whether one area is directly affected by its neighbors or accounting for the correlation between contiguous areas. In network analyses, one is similarly concerned that the outcome for one actor will be related to those for the other actors who influence her or him. In theory, each actor might directly or indirectly influence each other actor, which may lead to a complex correlation structure. Network data may be used to model such dependence though the construction of explanatory variables summarizing the level of the outcome among ones peers or in models with explicit error terms through the modeling of correlation among errors. ${ }^{12-18}$ In this paper, we focus on models that include peer variables of outcomes as opposed to error terms as they generalize more naturally to binary and other noncontinuous outcomes for which standard models do not have an error term. However, neither type of model allows peer effects to depend on the position of an actor in the network and so statistical methods for examining the hypotheses involving network strength modification of peer effects are lacking. We address this gap by developing statistical methodology for accommodating network strength (or other measure of structural importance) in the diffusion of a medical technology across a novel dynamic network of all US hospitals. After developing the general methodology, we apply it to the diffusion of implantable cardioverter defibrillators (ICDs) to provide a detailed illustration of the utility and scope of the methodology.

Evidence exists of associations of observed traits (or "phenotypes") among groups of individuals such as obesity ${ }^{19}$ and smoking, alcohol, or drug use. ${ }^{20,21}$ However, studies in which organizations are the nodes of the network are more elusive, although there are some exceptions in the healthcare domain. ${ }^{22-26}$ Prior studies involving network methods other than peer effect modeling in health care have tended to be cross sectional ${ }^{23,27-30}$ and so estimate concurrent associations. In contrast, we focus on longitudinal modeling.

In Section 2, the key ICD healthcare delivery measures, the control predictors, and the construction of the US hospital network for modeling the diffusion of ICDs are described. In Section 3, we develop a base peer effect model and derive several theoretical properties (derivations in the Appendix) to provide the basis for Section 4, in which we develop new models to account for the complexities of ICD diffusion by allowing peer-effect modification by network strength and simultaneous estimation of multiple peer effects. Estimation methods, including the use of hierarchical modeling to account for the complex correlation structure, are described in Section 5. Results of the ICD analyses appear in Section 7, and the paper concludes in Section 8.

\section{I DIFFUSION OF IMPLANTABLE CARDIOVERTER DEFIBRILLATOR (ICD) THERAPY}

A hospital implanting ICDs is termed an implanting or "capable" hospital, while a hospital implanting no ICDs is termed a referral or "noncapable" hospital. We are interested in peer effects (also termed social influence and contagion) of hospitals' capability to perform ICD procedures ("ICD capability"), the number of implants performed by the hospital 
if ICD capable, and the number of implants their patients received if ICD noncapable (these implants are necessarily performed at another hospital). We are also interested in the crossed effects: does the level of peer hospitals' ICD utilization predict the focal hospital's ICD capability and conversely does peer hospitals' ICD status predict the focal hospital's level of ICD utilization.

The ICD information for patients and hospitals was determined from the National Cardiovascular Disease Registry of patients who underwent ICD therapy for primary prevention from 2007 to 2011 . The annual number of ICDs for a noncapable hospital is made up of patients who received an ICD and whose plurality physician - the physician they encountered the most ${ }^{31}$-is one of the physicians attributed to that hospital in that year. Physician attribution to hospitals is based on their office location, if in the hospital, or where the majority of their patients are admitted, if their office is not located in a hospital. ${ }^{32}$

The ICD registry is the sole determinant of the ICD measures and forms the dependent variables for each analysis. It records all of the ICDs delivered to patients in the US, providing a significant advantage over data sets defined for subpopulations of patients such as those with certain demographics, living in a certain region, or who attended a certain hospital. A key point is that the measurement of the ICD measures for each hospital is a completely separate operation and involves an entirely different data set from the construction of the hospital network described in Section 2.1.

\section{1 | US cardiology care hospital network}

The network of hospitals is formed using US Medicare data. Because Medicare is the primary insurer across the US of persons over the age of 65 , the resulting networks are nationwide. We considered the claims most relevant to the diffusion of ICD adoption, ICD retention, and level of ICD utilization between hospitals to be those for patients who had a diagnosis of cardiovascular disease (arrhythmia, congestive heart failure, coronary heart disease, or peripheral vascular disease) and that took place in a hospital (captured by Medicare Part A claims) or that were for ambulatory (outpatient) care at a physician office (captured by Medicare Part B claims). ${ }^{33}$ In order to ensure that each patient's trajectory through the health system could be tracked in its entirety over the year, we required patients to have 12 months continuous Parts A and B coverage and excluded patients who had Medicare Advantage. Because Medicare Advantage is a form of private insurance whose claims are not included in the Medicare data, patients with Medicare Advantage will likely have gaps in their sequence of claims. Therefore, we are unable to identify all of the hospitals that provided care to a Medicare Advantage patient and thus the cotreatment of the patient across hospitals ("patient-sharing") would be unevenly undercounted if we incorporated these patients.

After extracting the relevant claims, we identified the physicians with whom patients had clinical encounters for which a cardiovascular disease diagnosis was on the insurance claim. A patient-hospital bipartite network is then obtained through aggregation of counts of physician patient sharing according to the attribution of the physicians who billed for them to hospitals. Thus, if a physician attributed to hospital A shares a patient with a physician attributed to hospital B, A and B have an edge in the network. The rationale for constructing the network using this bipartite approach is that we hope to capture the extent to which a patient is referred by a physician in hospital A to a physician in hospital B, potentially capturing information and knowledge sharing between the physicians and their hospitals, despite not having direct patient referral data at hand. The network is evaluated annually allowing for the evolution of relationships between hospitals over time. Because the ties are defined and evaluated for each pair of hospitals, we have complete "sociocentric" network data (the relationship is measured for each pair of hospitals) in each year. The approach is formalized below.

Let $S_{i j t}$ denote the set of patients who had at least one physician encounter associated with hospital $i$ and at least one physician encounter associated with hospital $j$ during year $t, m_{i j t}$ the cardinality of $S_{i j t}$, and $z_{i k t}$ the number of encounters with a cardiovascular disease diagnosis that patient $k$ in $S_{i j t}$ had with physicians in hospital $i$. The quantity $z_{i k t}$, or transformation thereof, is critical to the construction of the hospital network. For example, two types of edge weights between hospitals $i$ and $j$ in year $t$ are defined as follows:

$$
\begin{gathered}
a_{i j t}^{\prime}=\sum_{k \in S_{i j t}} I\left(z_{i k t}>0\right) I\left(z_{j k t}>0\right), \\
a_{i j t}^{\prime \prime}=\sum_{k \in S_{i j t}}\left(z_{i k t} z_{j k t}\right)^{1 / 2},
\end{gathered}
$$


where $I$ (event) denotes the indicator function equalling 1 if "event" is true and 0 otherwise. In Equation (1), $a_{i j t}^{\prime}$ represents the number of distinct patients with physician encounters at hospitals $i$ and $j$, while in Equation (2), $a_{i j t}^{\prime \prime}$ evaluates the geometric mean of the number of encounters patient $k$ had with hospitals $i$ and $j$ in projecting the (weighted) bipartite patient-hospital network to a unipartite hospital network. The square-root transformation in Equation (2) is motivated by the heuristic that given a fixed total number of visits there is more opportunity for influence to transmit between two hospitals if the patient visits them an equal number of times. We use $a_{i j t}=a_{i j t}^{\prime \prime}$ as it captures the notion that each time a patient transitions between hospitals there may be transmission and receipt of information with influence possibly being imparted between those hospitals. The matrix $\boldsymbol{A}_{t}$ with $i j$ th element $a_{i j t}$ is referred to as the adjacency matrix of the network at time $t$ and is the basis for modeling peer effects.

\section{3 | MODELING AND CHARACTERIZING PEER EFFECTS}

In this section, we develop the base peer-effects model and derive its key properties to establish the foundation for the innovative extensions to this model that are developed in Section 4 to model ICD diffusion. In the following, the term "ego" is used interchangeably with "focal" to refer to the actor being modeled (ie, that is subject to influence from others), while "alter" is similarly used interchangeably with "peer" when referring to actors having network ties with the ego (ie, the actors imparting influence). Let $\boldsymbol{W}_{t}=\left[w_{i j t}\right]$ be an $n \times n$ matrix whose $i j$ th element represents the influence actor $j$ exerts on actor $i$ in time period $t=1, \ldots, T$. In general, $\boldsymbol{W}_{t}$ is constrained so that

1. $w_{i j t} \geq 0$ : nonnegative weights.

2. $w_{i i t}=0$ : no self-influence.

3. $\sum_{j} w_{i j t}=1$ : weights give relative influences ( $\boldsymbol{W}_{t}$ is row-stochastic).

Network data may be used to model dependence though the construction of explanatory variables summarizing the level of the outcome among ones peers. Deciding how to use network data to construct $\boldsymbol{W}_{t}$ is an important step in the application of any network-based model of peer effects. ${ }^{16}$ A natural choice for $\boldsymbol{W}_{t}$ and the specification assumed for the ICD diffusion problem is as the row-stochastic counterpart of $\boldsymbol{A}_{t}$, the adjacency matrix of the network at time $t$. This specification assumes that influence is additive and is unaffected by whether or not common alters of an ego are alters of one another. As noted in Section 8, there are several extensions that could be applied to $\boldsymbol{W}_{t}$ and the modeling of peer effects beyond those considered in this paper.

Let $S_{i t}=\sum_{j=1}^{n} a_{i j t}$ be the sum of the edge weights involving actor $i$ at time $t$, a quantity known as actor $i$ 's "strength." For simplicity and for consistency with our motivating problem, we assume that the network is undirected so that $\boldsymbol{A}_{t}$ is symmetric. For $j \neq i$, it follows that $w_{i j t}=a_{i j t} / S_{i t}$ if $S_{i t}>0$ and $w_{i j t}=1 /(n-1)$ if $S_{i t}=0$ and that $\boldsymbol{W}_{t}$ is not symmetric whenever there is variation in $S_{i t}$ across $i$. Therefore, actors are assumed to be influenced by a given alter in proportion to the weight of the network tie they share or, if they have no alters, to be equally influenced by all other actors in the network, thereby adjusting for regression toward the mean. An actor with a single alter is wholly influenced by that actor, whereas an actor connected to all others is equally influenced by each. If isolates existed in the network (there are none in any year of the US hospital network), the binary variable $I\left(S_{i t}>0\right)$ may be included as a predictor. When $a_{i j t}$ is binary (eg, $i$ and $j$ share a patient vs not), $S_{i t}$ is the degree of actor $i$. As noted in Section $1, S_{i t}$ may be any measure of structural importance or prominence in the network, although in this paper we focus on strength alone. There is also no requirement that $\boldsymbol{W}_{t}$ be based solely on $\boldsymbol{A}_{t}$. For example, if it was felt that the strength of an alter affected the extent to which they imparted influence on the ego, the jth column of $\boldsymbol{A}_{t}$ could be weighted by $S_{j t}$ prior to forming $\boldsymbol{W}_{t}$. Additional extensions abound if the general assumptions defining $\boldsymbol{W}_{t}$ are relaxed; for example, relaxing condition 1 allows negative influences such as enemies as opposed to friends (beyond the scope of this paper) while relaxing condition 3 is akin to the innovative extension developed in Section 4.1.

Let $Y_{i t}$ and $\boldsymbol{X}_{i t}$ denote the outcome and a vector of $p$ covariates (including 1 for the intercept) for actor $i$ at time $t . \boldsymbol{Y}_{t}$ and $\boldsymbol{X}_{t}$ are the corresponding networkwide quantities; $\boldsymbol{X}_{i t}^{T}$ is the $i$ th row of the $n \times p$ matrix $\boldsymbol{X}_{t}$. Then define $\boldsymbol{W} \boldsymbol{Y}_{t}=\boldsymbol{W}_{t} \boldsymbol{Y}_{t}$ to be the vector whose $i$ th element, $W Y_{i t}=\left[\boldsymbol{W}_{t} \boldsymbol{Y}_{t}\right]_{i}=\sum_{j \neq i} w_{i j t} Y_{j t}$, is the weighted average of $\boldsymbol{Y}$ for actor $i$ 's peers. (The short-hand notation $\boldsymbol{W} \boldsymbol{Y}_{t}$ is favored over the hold-out mean, $\overline{\boldsymbol{Y}}_{(i) t}$, because it makes the involvement of the network explicit.) We favor a network influence construction in which $W Y_{i t}$ is the peer variable for actor $i$ over a dyad-level 
analysis ${ }^{19}$ with separate dyadic observations on $i$ for each $j \neq i$ because the dyadic equations with individual $i$ as subject are not compatible across $j .{ }^{34}$

We use a linear specification for the core model to aid interpretation and result derivation. Our core model is then the discrete-time dynamical system or Markov transition model:

$$
Y_{i t}=\beta_{1} Y_{i(t-1)}+\boldsymbol{\beta}_{2}^{T} \boldsymbol{X}_{i(t-1)}+\alpha_{1} W Y_{i(t-1)}+\epsilon_{i t}
$$

where $\boldsymbol{\beta}=\left(\beta_{1}, \boldsymbol{\beta}_{2}^{T}\right)^{T}$ is a vector of regression parameters, $\alpha_{1}$ is the peer effect, and $\epsilon_{i t}$ is the independent error or disturbance assumed to have mean 0 and variance $\sigma^{2} .{ }^{35-38}$ The model has the same structure as a time-space recursive model. ${ }^{39}$ The time between observations $t-1$ and $t$ is assumed to be sufficiently small that any change in the outcome of actor $i$ has yet to impact those of actors $j \neq i$, avoiding endogenous feedback. Conditioning on $Y_{i(t-1)}$ in Equation (3) imposes the Markov (or AR(1)) structure with the strength of the serial correlation quantified by $\beta_{1}$. To allow peer influence to operate through covariates, the alter-weighted covariate $\boldsymbol{W} \boldsymbol{X}_{i(t-1)}$ may be added to Equation (3) yielding the linear-in-means model ${ }^{40}$ under which $Y_{i t}$ depends on alter covariates as well as $\boldsymbol{X}_{i(t-1)}$. An alternative to the model in Equation (3) arises by assuming that $\epsilon_{i t}$ is related through the network to $\left\{\epsilon_{j(t-1)}\right\}_{j \neq i}$, the lagged error terms of an actors peers, as opposed to assuming that network dependence directly acts on outcomes. Models with both autocorrelated outcomes and autocorrelated errors have also been considered. ${ }^{37,41}$ In this paper, we take Equation (3) to be our base model as it generalizes more easily to binary outcomes, the form of the outcome for the adoption of a technology.

Under Equation (3), $\alpha_{1}$ is the change in $E\left[Y_{i t} \mid \boldsymbol{Y}_{t-1}\right]$ due to a unit increase in $W Y_{i(t-1)}$, which arises under an intervention in which in period $t-1$ every alter undergoes a unit change in $Y$ or any other combination of changes such that $W Y_{i(t-1)}$ increases by 1 . If only a subset of actors are intervened on, $E\left[Y_{i t} \mid \boldsymbol{Y}_{t-1}\right]$ increases by $\alpha_{1}$ multiplied by the weighted size of the subset in relation to the total. The parameter $\alpha_{1}$ represents the effect one would estimate in a hypothetical experiment in which an actor is randomly assigned peers with a given $\boldsymbol{W} \boldsymbol{Y}_{t-1}$ and then followed. However, in the application in Section 2, the observational nature of the data inhibits a causal interpretation. Readers are referred to existing literature for a more detailed review of model-based approaches for estimating peer effects. ${ }^{35,42,43}$

To explore the implications of Equation (3) more deeply and to distinguish the interpretation of a peer effect from a standard regression coefficient, we consider a special case of Equation (3) that converges to an equilibrium distribution. If $\left|\beta_{1}+\alpha_{1}\right|<1$, then as $t \rightarrow \infty$ the model has a steady state distribution in which (see Appendix)

$$
Y_{i}=\tilde{\alpha}_{1} \overline{W Y}_{i}+\boldsymbol{X}_{i}^{T} \tilde{\boldsymbol{\beta}}+\epsilon_{i}, \quad i=1, \ldots, n,
$$

or equivalently:

$$
Y_{i}=\left(\boldsymbol{I}-\tilde{\alpha}_{1} W\right)^{-1} \boldsymbol{X}_{i}^{T} \tilde{\boldsymbol{\beta}}+\left(\boldsymbol{I}-\tilde{\alpha}_{1} \boldsymbol{W}\right)^{-1} \epsilon_{i}, \quad i=1, \ldots, n,
$$

where $\tilde{\alpha}_{1}=\alpha_{1} /\left(1-\beta_{1}\right)$ and $\tilde{\beta}=\beta_{2} /\left(1-\beta_{1}\right)$. This cross-sectional model is commonly known as a network autocorrelation model. ${ }^{44}$ If $\boldsymbol{I}-\tilde{\alpha}_{1} \boldsymbol{W}$ is nonsingular, the asymptotic variance of $\boldsymbol{Y}$ satisfies

$$
\operatorname{var}(\boldsymbol{Y} \mid \boldsymbol{X})=\sigma^{2}\left(\boldsymbol{I}-\tilde{\alpha}_{1} \boldsymbol{W}\right)^{-1}\left(\boldsymbol{I}-\tilde{\alpha}_{1} \boldsymbol{W}\right)^{-T},
$$

illustrating that the marginal variance of the model depends both on the error variance and the observed network.

To illustrate how the ego and alter covariates combine to form an association with the outcome, suppose that the $k$ th of $n / 2$ dyads contains actors $(i, j)=(2 k-1,2 k)$. Then $\boldsymbol{W}$ can be arranged in block diagonal form and Equation (5) reduces to

$$
\left(\begin{array}{c}
Y_{i} \\
Y_{j}
\end{array}\right)=\frac{1}{1-\tilde{\alpha}_{1}^{2}}\left(\begin{array}{cc}
1 & \tilde{\alpha}_{1} \\
\tilde{\alpha}_{1} & 1
\end{array}\right)\left(\begin{array}{c}
\boldsymbol{X}_{i}^{T} \\
\boldsymbol{X}_{j}^{T}
\end{array}\right) \tilde{\boldsymbol{\beta}}+\frac{1}{1-\tilde{\alpha}_{1}^{2}}\left(\begin{array}{cc}
1 & \tilde{\alpha}_{1} \\
\tilde{\alpha}_{1} & 1
\end{array}\right)\left(\begin{array}{c}
\epsilon_{i} \\
\epsilon_{j}
\end{array}\right)
$$

with $\left|\tilde{\alpha}_{1}\right|<1$ for $\boldsymbol{W}$ to be nonsingular. Therefore, the weighted average covariates $\tilde{\boldsymbol{X}}_{i}=\left(\boldsymbol{X}_{i}+\tilde{\alpha}_{1} \boldsymbol{X}_{j}\right) /\left(1+\tilde{\alpha}_{1}\right)$ and $\tilde{\boldsymbol{X}}_{j}=$ $\left(\tilde{\alpha}_{1} \boldsymbol{X}_{i}+\boldsymbol{X}_{j}\right) /\left(1+\tilde{\alpha}_{1}\right)$ combine the ego's and alter's covariates into a single overall predictor of $Y_{i}$ and $Y_{j}$, respectively, with regression coefficient $\breve{\boldsymbol{\beta}}=\tilde{\boldsymbol{\beta}} /\left(1-\tilde{\alpha}_{1}\right)$. The condition $\left|\tilde{\alpha}_{1}\right|<1$ implies that under the conditions for a steady-state distribution to exist, the effect of the ego's predictor, $\boldsymbol{X}_{i}$, must exceed the effect of $\boldsymbol{X}_{j}$, the peer covariate. 


\section{4 | METHODOLOGICAL EXTENSION: MODELING THE DIFFUSION OF IMPLANTABLE CARDIOVERTER DEFIBRILLATOR (ICD) THERAPY}

As noted in Section 1 models for peer effects have to date been limited in that they have not allowed for modification of the peer effect by the network strength of an actor. In this section, we extend Equation (3) to account for the complexities of the ICD network by allowing peer effect modification by the importance of the ego hospital's network strength (Section 4.1) and distinct effects for ICD capable and noncapable hospitals (Section 4.2). Before considering the network-based part of the model, we describe the control variable for the ICD status of nearby hospitals, in its own right an innovative feature of our analysis. Let $\boldsymbol{G}$ denote a matrix whose $i j$ th element, $g_{i j}$ for $i \neq j$, depends on the physical distance (eg, geodesic distance or driving time) between hospitals $i$ and $j$, denoted $d_{i j}$. Although $d_{i j}$ could depend on $t$ (eg, a hospital could relocate due to a merger or acquisition), we do not observe such events in our data. We define $g_{i j}=1 /\left(1+v d_{i j}\right)$ if hospital $j$ was among the $m$ closest to $i$ and $g_{i j}=0$, otherwise, where $v$ is a parameter that governs the rate of decrease in the value of $g_{i j}$ as a function of $d_{i j}$. As for $\boldsymbol{W}_{t}$ (see Section 3), the diagonal of $\boldsymbol{G}$ is a vector of 0 s and we row-standardize $\boldsymbol{G}$ to make it a row-stochastic matrix. The combination of geographic and network information in $\boldsymbol{A}_{t}$ and $\boldsymbol{G}$ can be thought of as forming a multilayered network. We include $\boldsymbol{G} \boldsymbol{Y}_{t}=\boldsymbol{G} \times \boldsymbol{Y}_{t}$ as a predictor in the peer-effects models to separate the effect of the ICD status of nearby hospitals from that of the peer effects evaluated on the US hospital network.

We set $v=1$ in the calculation of $g_{i j}$ and tried several values of $m$, including $m=1,5,10$, and 25 for the ICD analysis. The restriction to the $m$ closest hospitals in terms of geodesic physical distance increases the variability of $d_{i j}$ across the hospital dyads compared to if all hospitals are used. The correlation between $d_{i j}$ and $w_{i j t}$ ranges between 0.247 and 0.256 across the 5 years, which are sufficient low to ensure that the coefficients of predictors involving $\boldsymbol{W Y}$ (including interactions with network strength) are separately identifiable from predictors involving $\boldsymbol{G} \boldsymbol{Y}$. Because the estimates of the predictors involving network peer-effect measures for the models described in Section 4.1 onward were only trivially affected by $m$ over this range, results are only presented for the $m=10$ case.

\section{1 | Peer effect modification}

The extension of Equation (3) to allow peer effect modification by the importance of the ego hospital's position in the network, herein measured by their network strength, and account of the impact of physically close hospitals is given by

$$
Y_{i t}=\beta_{1} Y_{i(t-1)}+\boldsymbol{\beta}_{2}^{T} \boldsymbol{X}_{i(t-1)}+\beta_{3} S_{i(t-1)}+\left(\alpha_{1}+\alpha_{2} S_{i(t-1)}\right) W Y_{i(t-1)}+\gamma_{1} G Y_{i(t-1)}+\epsilon_{i t} .
$$

All predictors are lagged to year $t-1$ to avoid the possibility that the covariates in year $t$ could be a consequence of the outcome for hospital $i$ in year $t$ (reverse causality). Because $S_{i(t-1)} W Y_{i(t-1)}=S_{i(t-1)} \sum_{j \neq i} w_{i j(t-1)} Y_{j(t-1)}=\sum_{j \neq i} a_{i j(t-1)} Y_{j(t-1)}$ with $S_{i(t-1)}=\sum_{j \neq i} a_{i j(t-1)}$, the $i$ th row-sum of $\boldsymbol{A}_{(t-1)}, \alpha_{2}$ corresponds to the difference in the effect of increasing the total number of peer hospitals that are ICD capable (multiplication by strength reverses the row-standardization operation that makes $\boldsymbol{W}_{t-1}$ row stochastic). Because $W Y_{i(t-1)}$ is volume independent, we think of $\alpha_{1}$ as a main effect and $\alpha_{2}$ as the modification of the peer-effect due to its network strength. If $S_{i(t-1)}$ is centered by its mean, $\alpha_{1}$ represents the peer effect for a hospital having average strength in the network. In general, $\alpha_{2}$ is the change in the peer effect of peer hospital ICD exposure under a unit change in $S_{i(t-1)}$. The peer effect profile is represented by a plot of $\alpha_{1}+\alpha_{2} S_{i(t-1)}$ against $S_{i(t-1)}$. Finally, $\gamma_{1}$ is interpreted as the effect of a one-unit increase in the level of ICD adoption by the hospitals whose location is close to that of hospital $i$.

\subsection{ICD capability, implant volume, and referral volume peer variables}

A further extension of Equation (3) allows for peer hospital influence to vary with the ICD capability status of both the ego-hospital and its peer hospitals. Because ICD capability is one of the outcomes modeled, to avoid notational confusion we use the variable $Q$ to denote ICD capability ( $1=$ capable, $0=$ noncapable). Therefore, hospital $i$ 's ICD capability in time period $t$ is given by $Q_{i t}$ and hospital $i$ 's exposure to ICD capable hospitals during $t$ is given by $W Q_{i t}$ with vector counterpart $\boldsymbol{W} \boldsymbol{Q}_{t}$. We use $Z_{i t}$ and $W Z_{i t}$ to denote hospital $i$ 's number of ICD procedures and the ICD utilization of its peer hospitals in time period $t$, respectively. 
We now define the weight matrices and peer hospital predictors used in the ICD capability and utilization models. Let $S_{i t}^{\mathrm{imp}}=\sum_{j \neq i}^{n} Q_{j t} a_{i j t}$ and $S_{i t}^{\text {ref }}=\sum_{j \neq i}^{n}\left(1-Q_{j t}\right) a_{i j t}$ denote the network-weighted sums of ICD capable ("imp" = implanting) and ICD noncapable ("ref" = referral) peer hospitals, respectively. The corresponding weight matrices, $\boldsymbol{W}_{t}^{\mathrm{imp}}$ and $\boldsymbol{W}_{t}^{\text {ref }}$, are then given by

$$
\begin{aligned}
w_{i j t}^{\mathrm{imp}} & =Q_{i t} a_{i j t} / S_{i t}^{\mathrm{imp}} \text { if } S_{i t}^{\mathrm{imp}}>0 \text { and } 0 \text { otherwise and } \\
w_{i j t}^{\mathrm{ref}} & =Q_{i t} a_{i j t} / S_{i t}^{\mathrm{ref}} \text { if } S_{i t}^{\mathrm{ref}}>0 \text { and } 0 \text { otherwise, }
\end{aligned}
$$

respectively. Likewise, the peer hospital predictors are obtained by multiplying $\boldsymbol{W}_{t}^{\mathrm{imp}}$ and $\boldsymbol{W}_{t}^{\text {ref }}$ by $\boldsymbol{Z}_{t}$ to obtain $\boldsymbol{W} \boldsymbol{Z}_{t}^{\mathrm{imp}}$ and $\boldsymbol{W} \boldsymbol{Z}_{t}^{\text {ref }}$.

The overall weight matrix $\boldsymbol{W}_{t}$ is related to $\boldsymbol{W}_{t}^{\mathrm{imp}}$ and $\boldsymbol{W}_{t}^{\mathrm{ref}}$ by:

$$
S_{i t} w_{i j t}=S_{i t}^{\mathrm{imp}} w_{i j t}^{\mathrm{imp}}+S_{i t}^{\mathrm{ref}} w_{i j t}^{\mathrm{ref}},
$$

implying that

$$
w_{i j t}=W Q_{i t} w_{i j t}^{\mathrm{imp}}+\left(1-W Q_{i t}\right) w_{i j t}^{\mathrm{ref}},
$$

as $W Q_{i t}=S_{i t}^{\mathrm{imp}} / S_{i t}$ is the proportion of hospital $i$ 's patients shared with ICD capable hospitals in year $t$. The expansion of $w_{i j t}$ in Equation (9) motivates the inclusion of $W Q_{i t}$ along with the peer variables $\boldsymbol{W} \boldsymbol{Z}_{t}^{\text {imp }}$ and $\boldsymbol{W} \boldsymbol{Z}_{t}^{\text {ref }}$, which capture the ego hospital's exposure to high volume peer implant and referral hospitals, respectively, as predictors in the ICD capability and utilization models.

\section{3 | Statistical outcome model for ICD capability status}

We model $Q_{i t}$ using two hierarchical logistic regression models that condition on $Q_{i(t-1)}=q$, where $q=0$ represents ICD noncapable and $q=1$ represents ICD capable. All predictors other than the number of cardiovascular patients attributed to the ego hospital, which controls for the number of patients potentially eligible for an ICD, are lagged by a year in order to isolate the effect of the peer hospital's influence on the ego hospital. Therefore, the model has the form:

$$
Q_{i t} \mid Q_{i(t-1)}=q, \operatorname{HRR}_{i(t-1)}=h, \eta_{h q}, \lambda_{q i} \sim \operatorname{Bernoulli}\left(\pi_{i t}(q, h)\right)
$$

where $\pi_{i t}(q, h)=E\left[Q_{i t} \mid Q_{i(t-1)}=q, \operatorname{HRR}_{i(t-1)}=h, \eta_{h q}, \lambda_{q i}\right]$,

$$
\begin{aligned}
\operatorname{logit}\left(\pi_{i t}(q, h)\right)= & \beta_{0 q}+\beta_{1 q} X_{i(t-1)}+\alpha_{1 q} W Q_{i(t-1)}+\alpha_{2 q} W Z_{i(t-1)}^{\mathrm{imp}}+\alpha_{3 q} W Z_{i(t-1)}^{\mathrm{ref}} \\
& +\left(\beta_{2 q}+\alpha_{4 q} W Q_{i(t-1)}+\alpha_{5 q} W Z_{i(t-1)}^{\mathrm{imp}}+\alpha_{6 q} W Z_{i(t-1)}^{\mathrm{ref}}\right) S_{i(t-1)} \\
& +\gamma_{1 q} G Q_{i(t-1)}+\gamma_{2 q} G Z_{i(t-1)}^{\mathrm{imp}}+\gamma_{3 q} G Z_{i(t-1)}^{\mathrm{ref}}+\eta_{q h}+\lambda_{q i},
\end{aligned}
$$

$\operatorname{HRR}_{i(t-1)}$ is the health referral region (HRR) that hospital $i$ is in during time period $t-1, \eta_{q h} \sim \operatorname{Normal}\left(0, \omega_{q}^{2}\right)$ is the random effect for $\operatorname{HRR} h(h=1, \ldots, H)$, and $\lambda_{q i} \sim \operatorname{Normal}\left(0, \tau_{q}^{2}\right)$ is a random effect for hospital $i$. The dependence of the parameters on $q$ allows for dependence of the effects of the predictors on the ICD capability of the ego hospital at $t-1$. The multiple extensions to the model make closed-form derivations that emulate Section 3 for Equation (10) challenging. However, Section 3 provides a helpful basis for peer effect interpretation even in this more complex situation.

The adoption $(q=0)$ model estimates associations between the predictors and the likelihood of adopting ICD capability during the following year. Conversely, the ICD continuation $(q=1)$ model estimates associations between the predictors and the likelihood that the hospital remains ICD capable over the next year. For example, $\alpha_{10}>0$ implies that having a high proportion of ICD capable peers is associated with adoption of ICD capability while $\alpha_{11}>0$ implies that having a high (network weighted) proportion of capable peers is associated with a hospital remaining ICD capable. The computation of the peer hospital predictors for these models is illustrated in Figure 1. 


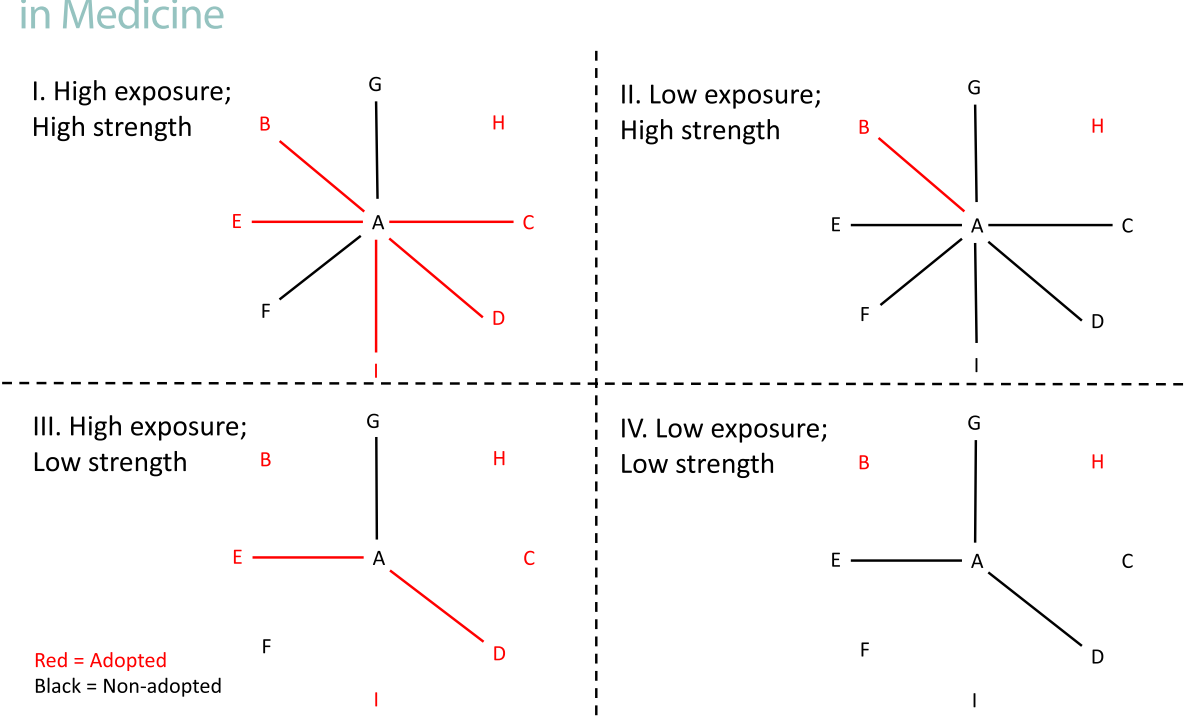

F I G U R E 1 Schematic illustrating the four combinations of high vs low rate of adoption crossed with strong vs low network strength; for simplicity the edge weights are equal and so each hospitals network strength equals its degree in the network. Red nodes and edges indicate hospitals that have already adopted while black indicates nonadopters. We hypothesize that Scenario I is the most conducive for the hospital A, the ego, to be influenced to adopt and that the contrast of scenario I vs scenario II yields a positive peer effect while the contrast of scenario III vs scenario IV yields a negative peer effect [Color figure can be viewed at wileyonlinelibrary.com]

Because a hospital may be both ICD capable and noncapable during the study period, it may contribute to both the estimation of the adoption and continuation models. Therefore, parameters may be shared or correlated across the adoption and continuation models; for example, nonzero correlations may be allowed between the elements of $\left\{\left(\eta_{0 h}, \eta_{1 h}\right)\right\}_{h=1, \ldots, H}$ or between the elements of $\left\{\left(\lambda_{0 i}, \lambda_{1 i}\right)\right\}_{i=1, \ldots, n}$.

\section{4 | Statistical outcome model for implant and referral volume}

If $Q_{i t}=1, Z_{i t}$ is the number of ICDs implanted by hospital $i$ while if $Q_{i t}=0, Z_{i t}$ is the number of patients attributed to hospital $i$ who received an ICD elsewhere. To ensure that the ego hospital ICD count at the current time had the same interpretation as in the prior year, the peer effect analyses of the number of ICDs implanted or referred are restricted to hospital years for which the ego hospital had the same ICD capability as in the prior year. Therefore, we model $Z_{i t}$ as a Poisson random variable conditional on $Q_{i t}=Q_{i(t-1)}=q$ with a $\log \operatorname{link}$ and an offset $\log \left(m_{i t}\right)$, where $m_{i t}$ is the number of patients suffering from cardiovascular disease affiliated with hospital $i$ in time period $t$. If $q=1, Z_{i t}$ is the number of ICDs implanted by hospital $i$ while if $q=0, Z_{i t}$ is the number of ICDs received (at other hospitals) by the patients of hospital $i$ 's physicians. The models are given by:

$$
Z_{i t} \mid Q_{i t}=Q_{i(t-1)}=q, \operatorname{HRR}_{i(t-1)}=h, \phi_{h q}, \theta_{q i} \sim \operatorname{Poisson}\left(\mu_{i t}(q, h)\right),
$$

where

$$
\begin{aligned}
\log \left(\mu_{i t}(q, h)\right)= & E\left[Z_{i t} \mid Q_{i t}=Q_{i(t-1)}=q, \mathrm{HRR}_{i(t-1)}=h, \eta_{h q}, \theta_{q i}\right] \\
= & \beta_{0 q}+\beta_{1 q} Z_{i(t-1)}+\beta_{2 q} X_{i(t-1)}+\alpha_{1 q} W Q_{i(t-1)}+\alpha_{2 q} W Z_{i(t-1)}^{\mathrm{imp}}+\alpha_{3 q} W Z_{i(t-1)}^{\mathrm{ref}} \\
& +\left(\beta_{3 q}+\alpha_{4 q} W Q_{i(t-1)}+\alpha_{5 q} W Z_{i(t-1)}^{\mathrm{imp}}+\alpha_{6 q} W Z_{i(t-1)}^{\mathrm{ref}}\right) S_{i(t-1)}, \\
& +\gamma_{1 q} G Q_{i(t-1)}+\gamma_{2 q} G Z_{i(t-1)}^{\mathrm{imp}}+\gamma_{3 q} G Z_{i(t-1)}^{\mathrm{ref}}+\phi_{q h}+\theta_{q i}+\log \left(m_{i t}\right),
\end{aligned}
$$

$\phi_{q h} \sim \operatorname{Normal}\left(0, v_{q}^{2}\right)$ and $\theta_{q i} \sim \operatorname{Normal}\left(0, \sigma_{q}^{2}\right)$ are the random effects for HRR $h$ and hospital $i$, respectively, in the implant $(q=1)$ and referral volume $(q=0)$ models. 
The model in Equation (11) includes two peer variables (implant volume $W Z_{i(t-1)}^{\mathrm{imp}}$, referral volume $W Z_{i(t-1)}^{\mathrm{ref}}$ ) and their modification by the network strength of the ego hospital's network strength, $S_{i(t-1)}$, corresponding to their strength (ie, their total shared cardiology care). If $\alpha_{60}>0$, then the number of ICDs received by patients of peer ICD noncapable hospitals in year $t-1$ has a positive association with the number of ICDs received by the patients of ICD noncapable $(q=0)$ ego hospitals in year $t$ while $\alpha_{61}>0$ implies a positive association between noncapable peer hospital referral volume and equipped ego hospital implant volume in year $t$, all else equal.

\section{5 | MODEL BUILDING AND ESTIMATION}

Models were developed in three major steps: determining which ego covariates to include, selecting the prominent peer variables, and evaluating which peer variables interact with the network strength of the ego. In all statistical models, we adjust for year, the number of cardiovascular disease patients attributed to the hospital (patient volume), the number of physicians involved in shared cardiovascular disease patient care within the hospital (hospital size), the hospital's network strength, the distance to the 10 closest hospitals in physical distance and the associated rate of adoption $\left(G Q_{i(t-1)}\right)$ or level of utilization $\left(G Z_{i(t-1)}^{\mathrm{imp}}\right.$ and $\left.G Z_{i(t-1)}^{\mathrm{ref}}\right)$ of those hospitals, the fraction of shared cardiovascular disease patient care that is within the hospital (a quantity termed "fragmentation," 45 ), the lagged outcome and the ICD status of nearby hospitals. All predictors other than year and the number of cardiovascular disease patients attributed to the hospital are lagged. We also investigated using the ratio of peer total ICD referrals to total ICD implants as a predictor but found that it did not have a major association with ICD capability or level of utilization. With the exception of $G Y_{i(t-1)}$, which is explicitly represented, all of these predictors are included in $\boldsymbol{X}_{i t}$. Fragmentation is given by frag $i t=$ internal $_{i t} /\left(\right.$ internal $_{i t}+S_{i t}$ ), where internal ${ }_{i t}$ is the number of occasions a patient visited pairs of physicians both affiliated with hospital $i$ in year $t$. Recall that $S_{i t}$, the "strength" of hospital $i$, is the number of patients that visited a physician in hospital $i$ and a physician in another hospital.

In developing the peer hospital predictors for each model, we initially included the full set of peer ICD capability, implant volume, and referral volume variables and their interactions with the network strength of the ego. The nonsignificant peer variable interactions were judiciously removed until only significant peer variable interactions remained. The models that resulted were further simplified by removing nonsignificant peer variables that were not involved in interactions nor required for interpretive comparisons with other models. The rationale for this strategy is that there is a relatively small number of predictors in the model and our focus is on interpreting the results as opposed to finding the most parsimonious specification. Therefore, we are not concerned about including peer variable predictors in the final specification that are not significant. We assess model fit using methods for binary regression models and for linear regression models. These included using Hosmer-Lemeshow tests for binary outcomes and residual plots for continuous outcomes comparing fitted and actual values of the outcome and the fitted residuals with the values of each predictor (including predictors involving peer variables). The difference in model fit between competing models was evaluated using the deviance model-fit statistic. Although the models are complex with the peer variables being evaluated using intense data operations, the fact that the predictors are lagged allows traditional model fit assessments and criteria such as these to be interpreted in the usual manner. We did not identify any serious concerns regarding model fit across the estimated models.

Because the analyses only consider lagged peer variables, maximum (or restricted maximum) likelihood estimation of hierarchical generalized linear models for $Q_{i t}$ and $Y_{i t}$ yield consistent parameter estimates. This is seen from the assumed independence of the random effects, which implies $Q_{i t}, \lambda_{i} \perp Q_{j t}, \lambda_{j} \mid Q_{i(t-1)}, W Q_{i(t-1)}$ and $Y_{i t}, \theta_{i} \perp Y_{j t}, \theta_{j} \mid Y_{i(t-1)}, W Y_{i(t-1)}$, $j \neq i$. Therefore, despite an ego's lagged outcome being a predictor of another hospital's outcome, the ego's outcome at $t$ is conditionally independent (given the predictors at $t-1$ ) of its peers' outcomes at $t$, avoiding the need for multivariate modeling of $\boldsymbol{Y}_{t}$.

\section{6 | SIMULATION STUDY: OPERATING CHARACTERISTICS OF THE EXTENDED PEER EFFECTS MODEL}

To confirm that the proposed estimation procedure is able to estimate the true values of the network-strength-modification of the peer-effects for the models in Equations (8), (10), and (11), we perform a simulation study. Because the peer-effects 

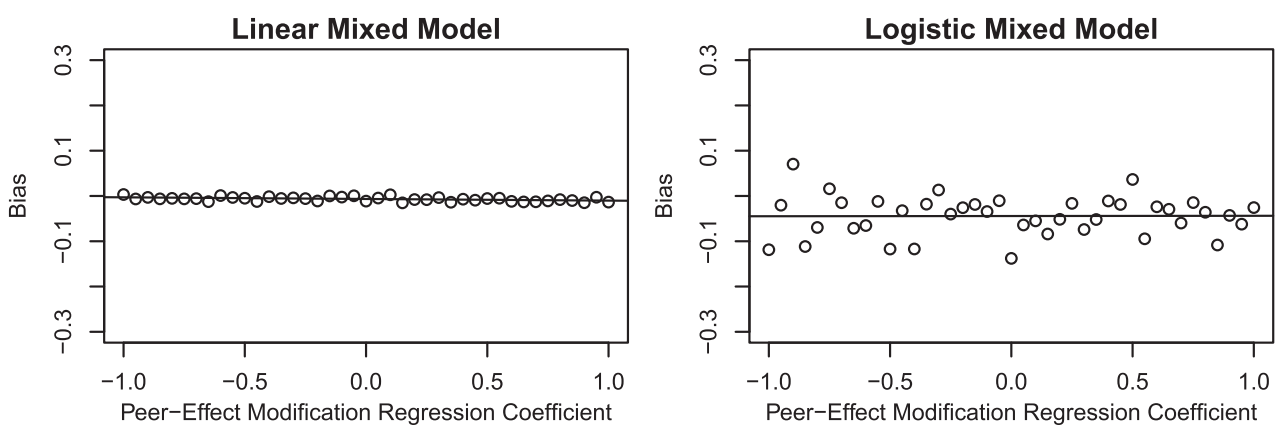

FIGURE 2 Bias, mean-squared-error (MSE), and coverage as a function of the $\alpha_{2}$, the regression coefficient of the network-strength-modified peer variable under Equation (8) and its logistic regression counterpart. These were evaluated using 1000 simulated data sets for each value of $\alpha_{2}$. The mean bias and coverage across the values of $\alpha_{2}$ are plotted
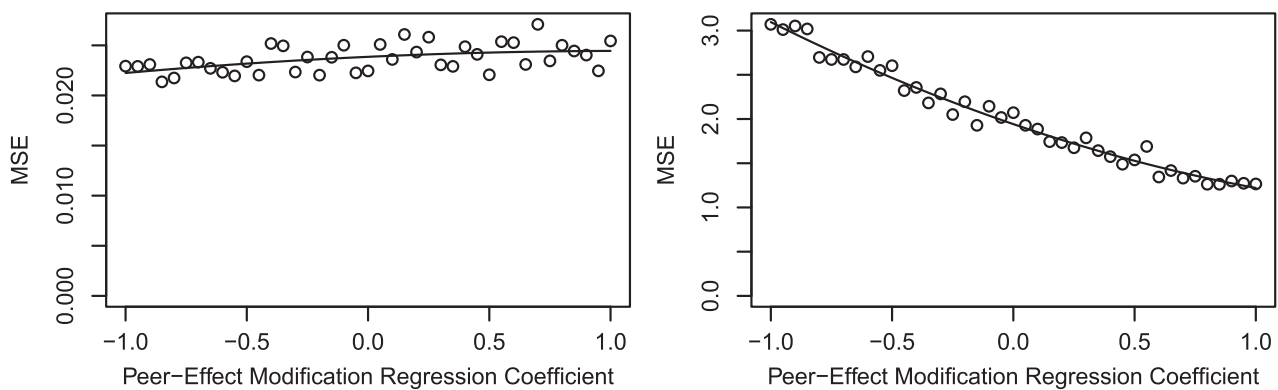
to show the closeness of the simulated means to the nominal values of 0 and 0.95 , respectively
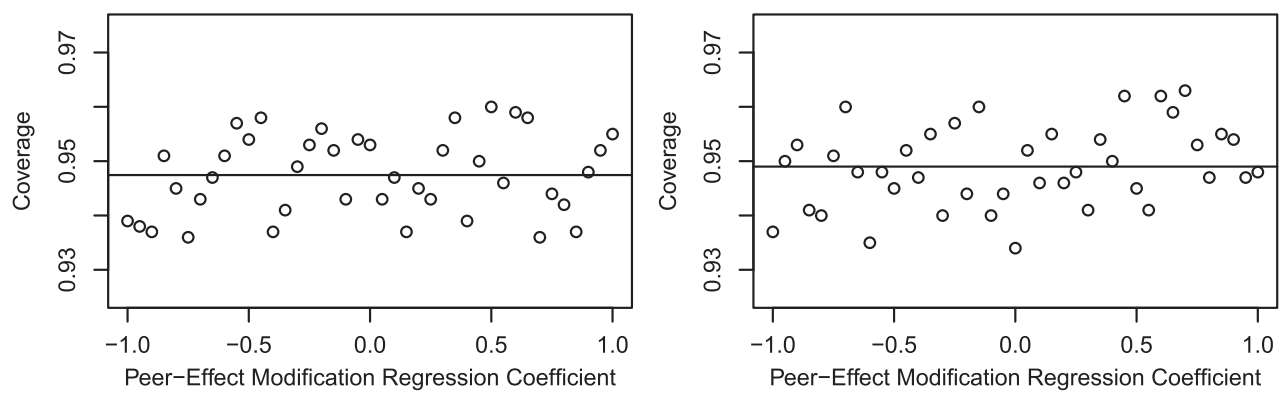

and their interactions enter the models additively, it suffices to show that the network-strength modification of the peer-effect for the model in Equation (8) and its hierarchical logistic regression counterpart for a binary outcome can be recovered. We performed a simulation study in which all model parameters other than $\alpha_{2}$ were fixed. All data elements other than the outcome variable were also fixed. A relatively small undirected network of $n=100$ actors over 5 years was considered to allow the simulation to be performed with many iterations relatively quickly. The simulated networks were random networks with density approximately 0.2 and were fixed across time (allowing change in the network had minimal impact). The weight matrix $\boldsymbol{W}$ was obtained by row-standardization of each simulated network. Without loss of generality, a single covariate is assumed; its effect may be viewed as the net effect of all covariates other than those involved in interactions with the peer variable predictor. Thus, $\boldsymbol{X}_{i(t-1)}$ is a $n$ by 2 matrix with an intercept and the covariate (coefficients $\beta_{0}$ and $\beta_{1}$, respectively) but as in Equation (8) the model also includes predictors for the lagged outcome and network strength (coefficients $\beta_{2}$ and $\beta_{3}$, respectively). Each of network strength, the covariate, the hospital random effects and the error term are assumed to have normal distributions. For the linear case, the outcome is conditionally normal whereas in the binary case the outcome is assumed to be related to the predictors via the logit function; for each observation a binary outcome was generated by evaluating whether a random uniform $(0,1)$ variable was less than the probability of the outcome. The values of $\left(\beta_{0}, \beta_{1}, \beta_{2}, \beta_{3}, \alpha_{1}, \tau^{2}, \sigma^{2}\right)$ were fixed at $(1,0.1,0.2,-1.5,0.25,1,2)$ while the value of $\alpha_{2}$ was varied from -1 to 1 with intervals of 0.05 . The bias, mean-squared-error (MSE), and coverage of the $95 \%$ confidence interval were then evaluated across the 1000 simulated data sets for each value of $\alpha_{2}$.

Under the hierarchical linear model, the operating characteristics of the estimation procedure are excellent (Figure 2). The bias is very close to 0 and shows no relation to $\alpha_{2}$, MSE is small, and coverage is close to the nominal 0.95 level. Under the hierarchical logistic model, a small negative bias is observed (average approximately -0.04), MSE is as expected much greater than for the linear model, and coverage is excellent. The small negative bias is indicative of the nonlinear nature 
of the logistic regression equation combined with the relatively small cluster size of 5 , which impacts the estimates of regression coefficients of terms like the network-based variables whose estimates are primarily determined from between individual variation. Similar results are obtained at other values of the model parameters and if $n$ is increased the estimators become more precise (MSE shrinks). Interestingly, MSE decreases as $\alpha_{2}$ increases under the hierarchical logistic regression model, whereas MSE is largely invariant to $\alpha_{2}$ under the corresponding hierarchical linear regression model. We attribute this result to the fact that the variance of Bernoulli distributed random variables depends on its mean, which at the values of the parameters used in the simulation is greatest when $\alpha_{2}$ is -1 . The same phenomena does not occur under the linear model as the conditional variance of an observation (or the residual variance) does not depend on its mean. In general, the simulation results imply that if the model is correctly specified then the maximum likelihood estimator of the parameter for the network-strength-modification of the peer-effect performs well under models with either linear or logistic forms. The fact that we obtain such encouraging results on a network that is smaller than the network used in the motivating application suggests that even better performance may be expected to hold for the analyses of that network (Section 7).

\section{7 | RESULTS OF US HOSPITAL NETWORK PEER EFFECT ANALYSES}

The outcome and network data include all hospitals with at least a single patient who received an ICD at that hospital or elsewhere over the period 2007-2011. Hospitals that closed were dropped from the data set in the years after closure. In total, 4734 unique hospitals contributed 23,066 observations of hospitals' ICD capability. The number of hospitals in the network ranged from 4520 to 4734 from 2007 to 2011 with 4496 hospitals present throughout. However, due to the use of lagged variables as predictors, only 18,332 observations were available to estimate the statistical models and the number of hospitals ranged from 4506 to 4638 implying even less variation in the relevant part of the peer network across time. Although changes in the network across time may impact network summary statistics such as strength, such variation aids estimation of the hierarchical models by increasing the amount of within hospital variation in the network summary measures and also possibly of the peer-hospital predictors. The inclusion of year as a predictor and hospital ID as a random effect in the statistical models accounts for general changes across time in the outcome distribution and optimally weights the extent to which within- and between-hospital variation in the peer predictors is used to identify their effects.

The distributions of both degree (number of ties to hospitals with strength $>0$ ) and strength are stable across the study period but noticeably right-skewed, especially strength (Table 1). The minimum degree is 1 due to the absence of isolates, while the maximum degree of 2359 reveals that the most connected hospital (the Memorial Herman Hospital System in Houston, Texas, USA) has ties with over 50\% of other hospitals in the US network). The number of patients shared conditional on sharing at least one patient increased from 83.2 to 98.8 over the 5 years.

Hospitals may have multiple transitions between ICD capable and ICD noncapable over the study period. The most common two-period state is ICD noncapable both years $(N=13,149)$ and the second is ICD capable both years $(N=4070)$. On $5.30 \%$ of occasions ( 736 out of 13,885 opportunities), an adoption of ICD capability and on $8.48 \%$ of occasions (377 out of 4447 opportunities) a deadoption of ICD capability occurred (Table 2).

The proportion of hospitals that were ICD capable jumped from 2007 to 2008 but remained flat thereafter (Table 3). The average number of patients at ICD noncapable hospitals who received ICDs declined from 2007 to 2008 and then stabilized while the average number of ICDs implanted by ICD capable hospitals had a nonmonotonic trajectory. The mean number of implants performed by ICD capable hospitals greatly exceeded the number received from other hospitals by patients of ICD noncapable hospitals.

The ego hospital control variables are highly correlated with the proportion of capable hospitals and the numbers of ICDs they implant, if capable, or the number received by the patients of their attributed physicians, if noncapable (Table 4). For example, the proportion of capable hospitals is 0.451 higher among ego hospitals with below median distance to their 10 closest neighbors and the correlations of the physical closeness measure with referral and implant ICD volume both exceeding 0.5. Thus, peer ICD capability appears to be negatively associated with ego hospital capability and the number of ICDs for which it is responsible. The sizable associations involving physical closeness illustrates the need to control for it in order to avoid confounding its effect with the network peer effects. 
TA B L E 1 Distribution of hospitals' degree and strength in the US hospital network

\begin{tabular}{lllllllll} 
Statistic & Year & Mean & SD & Minimum & LowerQ & Median & UpperQ & Maximum \\
Degree & 2007 & 217 & 238 & 1 & 50 & 137 & 313 & 2,411 \\
(distinct & 2008 & 213 & 234 & 1 & 50 & 135 & 305 & 2,359 \\
\hline hospitals & 2009 & 216 & 237 & 1 & 49 & 136 & 314 & 2,478 \\
with & 2010 & 216 & 237 & 1 & 49 & 136 & 314 & 2,478 \\
\hline shared & 2011 & 225 & 247 & 1 & 50 & 138 & 328 & 2,428 \\
patients) & Average & 218 & 239 & 1 & 50 & 136 & 315 & 2,431 \\
\hline Strength & 2007 & 18,086 & 28,429 & n/a & 1,391 & 6,130 & 22,990 & 279,668 \\
(distinct & 2008 & 19,573 & 31,017 & n/a & 1,404 & 6,486 & 25,074 & 324,177 \\
\hline patients & 2009 & 20,312 & 32,302 & n/a & 1,444 & 6,688 & 25,633 & 324,751 \\
shared) & 2010 & 20,312 & 32,302 & n/a & 1,444 & 6,688 & 25,633 & 324,751 \\
\hline & 2011 & 22,261 & 35,490 & n/a & 1,425 & 7,021 & 28,842 & 338,946 \\
\hline
\end{tabular}

Note: A total of 4734 hospitals contributed observations. A hospital's degree is the number of other hospitals it shared at least one patient with while strength (often referred to as weighted degree) is the total number of patients it shared with those hospitals. These correspond to $S_{i(t-1)}=\sum_{j \neq i} a_{i j(t-1)}$ when $\boldsymbol{A}_{t-1}$ is the adjacency or sociomatrix matrix of a binary and a weighted network, respectively. Because strength is a count of patients, minimum values less than 11 are indicated as n/a in accordance with the Center for Medicare and Medicaid's suppression rule.

\begin{tabular}{|c|c|c|c|}
\hline \multirow[b]{2}{*}{ Current ICD State } & \multicolumn{3}{|c|}{ New ICD State } \\
\hline & Noncapable & Capable & Total \\
\hline Noncapable & 13,149 & 736 & 13,885 \\
\hline Capable & 377 & 4,070 & 4,447 \\
\hline Total & 13,526 & 4,806 & 18,332 \\
\hline
\end{tabular}

TA B L E 2 Transition matrix of hospitals' ICD capability

\begin{tabular}{|c|c|c|c|c|c|c|}
\hline \multirow[b]{3}{*}{ Term } & \multirow{3}{*}{$\begin{array}{l}\text { Relative } \\
\text { Frequency }\end{array}$} & \multirow{3}{*}{$\begin{array}{l}\text { Proportion } \\
\text { Capable }\end{array}$} & \multicolumn{4}{|c|}{ \# ICDs by Status } \\
\hline & & & \multicolumn{2}{|c|}{ Noncapable } & \multicolumn{2}{|c|}{ Capable } \\
\hline & & & Mean & SD & Mean & SD \\
\hline \multicolumn{7}{|c|}{ Ego ICD capable (Nobs = 23,066 over 4734 hospitals) } \\
\hline No & 0.755 & 0 & 3.19 & 7.86 & $\mathrm{n} / \mathrm{a}$ & $\mathrm{n} / \mathrm{a}$ \\
\hline Yes & 0.245 & 1 & $\mathrm{n} / \mathrm{a}$ & $\mathrm{n} / \mathrm{a}$ & 32.1 & 30.2 \\
\hline \multicolumn{7}{|c|}{ Year (Nobs $=23,066$ over 4734 hospitals) } \\
\hline 2007 & 0.196 & 0.184 & 5.91 & 13.77 & 37.5 & 35.3 \\
\hline 2008 & 0.198 & 0.257 & 2.25 & 4.61 & 27.3 & 25.7 \\
\hline 2009 & 0.200 & 0.259 & 2.85 & 5.72 & 35.5 & 33.6 \\
\hline 2010 & 0.200 & 0.269 & 2.66 & 5.37 & 32.8 & 30.0 \\
\hline 2011 & 0.205 & 0.253 & 2.09 & 4.14 & 28.7 & 25.3 \\
\hline
\end{tabular}

TA B L E 3 Frequency distribution of discrete-valued characteristics and associated levels of hospitals' ICD capability and number of ICDs 
T A B L E 4 Distribution of continuous or ordinal-valued predictors and their unadjusted association with ICD capability and ICD utilization

\begin{tabular}{|c|c|c|c|c|c|c|}
\hline \multirow[b]{2}{*}{ Variable } & \multirow[b]{2}{*}{$\boldsymbol{N}$} & \multicolumn{2}{|c|}{ Distribution } & \multirow[b]{2}{*}{$\begin{array}{l}\text { Change } \\
\text { Pr(Capable) }\end{array}$} & \multicolumn{2}{|l|}{ Corr(\# ICDs) } \\
\hline & & Mean & SD & & Noncapable & Capable \\
\hline Ego \# ICDs & 23,066 & 10.3 & 20.6 & 0.501 & 1.000 & 1.000 \\
\hline \multicolumn{7}{|l|}{ Ego hospital controls } \\
\hline \# cardiovascular patients & 21,934 & 1,729 & 2,332 & 0.478 & 0.715 & 0.751 \\
\hline \# physicians & 22,663 & 62.6 & 86.5 & 0.467 & 0.594 & 0.567 \\
\hline Network strength $\left(\times 10^{-5}\right)$ & 23,066 & 0.204 & 0.323 & 0.462 & 0.562 & 0.591 \\
\hline (Physical) closeness & 23,066 & 80,729 & 136,445 & 0.451 & 0.527 & 0.533 \\
\hline Fragmentation & 23,066 & 0.27 & 0.175 & 0.343 & 0.353 & 0.244 \\
\hline \multicolumn{7}{|c|}{ Peer hospital exposure and control variables } \\
\hline Peer capability & 23,066 & 0.638 & 0.229 & -0.100 & -0.088 & -0.140 \\
\hline Peer mean referrals & 23,066 & 8.51 & 10.45 & 0.046 & 0.147 & 0.107 \\
\hline Peer mean implants & 23,066 & 45.6 & 23.8 & -0.021 & 0.124 & 0.075 \\
\hline Closeness capability & 23,066 & 0.634 & 0.256 & -0.062 & -0.066 & -0.106 \\
\hline Closeness mean referrals & 22,949 & 8.14 & 10.90 & 0.051 & 0.146 & 0.109 \\
\hline Closeness mean implants & 23,066 & 44.7 & 25.4 & 0.125 & 0.125 & 0.067 \\
\hline
\end{tabular}

Note: Change $\operatorname{Pr}($ Capable $)$ is the difference in the proportion of capable hospitals for hospitals above the median value of the left-hand-variable to those below the median value. The correlations are of the left-hand-variable with the total number of ICDs received by patients of noncapable and capable hospitals. Network strength, the number of distinct patients shared with other hospitals, is the sum of the weights on the network edges of a hospital to each of the other hospitals (edges have a value of 0 if the hospitals share no patients). Physical closeness is proportional to $g_{i j}=1 /\left(1+d_{i j}\right)$, where $d_{i j}$ is the geodesic distance in units of approximately 28 miles (this makes the longest straight-line distance across the continental US 100 units) between hospitals $i$ and $j \neq i$ averaged over the 10 closest hospitals to $i$.

\section{1 | Models of adoption of ICD capability status}

The parameter estimates for the logistic regression models regressing $Q_{i t}(1=$ capable, $0=$ noncapable $)$ on $W Q_{i(t-1)}$ and the other predictors for each value of $Q_{i(t-1)}$ are shown in Table 5. The highly positive and significant interaction effect of 2.659 , which reflects a progression from a negative to a positive peer ICD capability association across the range of values of strength (effect trajectory $-0.786+2.659 \times$ strength), in the adoption model is compelling. The 77.6'th percentile of hospital network strength (equal to 0.296) is the point at which the peer ICD capability association crosses 0 to become positive (Figure 3) while by the 95 'th percentile of network strength it equals 1.45 , corresponding to odds of 3.85 of adopting ICD capability. These results suggest that network strength quantifies the extent to which exposure to a high proportion of peer hospital adopters encourages or discourages adoption. The results may also reflect that stronger patient-sharing ties are a more reliable indicator of a true interhospital influence relationship than weaker patient-sharing ties. ${ }^{46}$ To depict the interaction effect in this and the remaining statistical models, Figure 3 displays the estimated peer association as a function of the ego hospital's strength.

The deviance model-fit statistic of the model in Equation (10) compared to the "baseline" model, the model without the two network peer variables, is 8.81 smaller $(p=0.0122)$ implying that the peer effects make a significant contribution to the model. However, the level of ICD-utilization for both capable and noncapable peer hospitals had minimal impact on adoption (these nonsignificant predictors were excluded from the final model so do not appear in Table 5), illustrating that the independent variation explained by peer hospitals is primarily due to the interactions between the network strength of the ego hospital and the ICD capability of its peer hospitals. The positive associations of nearby hospitals' ICD capability on adoption (log-odds $1.46, p<0.001$ ) are consistent with hospitals emulating other hospitals to compete for patients in their local market. When both lagged peer ICD capability and lagged closeness ICD capability are included in the model 
T A B L E 5 Estimated peer effect models for adoption (306 HRRs, 3720 hospitals, 12,716 observations) and continuation (305 HRRs, 1410 hospitals, 4418 observations) of ICD capability

\begin{tabular}{|c|c|c|c|c|c|c|}
\hline \multirow[b]{2}{*}{ Term } & \multicolumn{3}{|c|}{ Adoption } & \multicolumn{3}{|c|}{ Continuation } \\
\hline & Estimate & Z-stat & $p$-value & Estimate & Z-stat & $p$-value \\
\hline Intercept & -9.794 & -16.33 & 0.000 & 1.078 & 1.90 & 0.058 \\
\hline Year 2008 & 0.00 & & & 0.00 & & \\
\hline Year 2009 & -0.802 & -5.48 & 0.000 & 0.384 & 1.82 & 0.068 \\
\hline Year 2010 & -0.480 & -3.43 & 0.001 & 0.141 & 0.66 & 0.512 \\
\hline Year 2011 & -0.867 & -5.52 & 0.000 & -0.398 & -1.92 & 0.055 \\
\hline \# patients (1000s) & 0.460 & 6.50 & 0.000 & 0.048 & 0.73 & 0.464 \\
\hline Lag \# physicians (100s) & 0.793 & 4.74 & 0.000 & -0.214 & -1.87 & 0.061 \\
\hline Lag strength $\left(10^{5}\right)$ & -3.084 & -4.42 & 0.000 & -1.976 & -3.29 & 0.001 \\
\hline Lag (physical) closeness & 1.111 & 8.73 & 0.000 & 0.211 & 2.37 & 0.018 \\
\hline Lag fragmentation & 3.454 & 6.59 & 0.000 & 1.039 & 1.75 & 0.080 \\
\hline Lag \# ICDs (10s) & 0.607 & 8.35 & 0.000 & 0.822 & 10.09 & 0.000 \\
\hline Lag peer capable & -0.786 & -2.22 & 0.027 & -1.762 & -3.42 & 0.001 \\
\hline Lag peer capable*strength & 2.659 & 2.86 & 0.004 & 2.108 & 2.42 & 0.016 \\
\hline Lag closeness capable & 1.460 & 3.46 & 0.001 & 0.418 & 1.03 & 0.305 \\
\hline \multirow[t]{2}{*}{ Var(hospital) } & \multicolumn{3}{|l|}{$2.47 \pm 1.56$} & \multicolumn{3}{|l|}{$0.79 \pm 0.89$} \\
\hline & \multicolumn{3}{|l|}{$0.69 \pm 0.83$} & \multicolumn{3}{|l|}{$0.00 \pm 0.00$} \\
\hline
\end{tabular}

Note: The net peer ICD capability effects are given by $-0.786+2.659 \times$ strength and $-1.762+2.108 \times$ strength in the adoption and continuation models, respectively. The numbers in the parentheses in the left-most column quantify the unit change to which the estimated regression coefficients correspond. For example, the coefficients of terms involving strength are in units of $10^{5}$ shared patients in this and subsequent tables of results.

\section{ICD Adoption and Continuation Model Peer Effects}

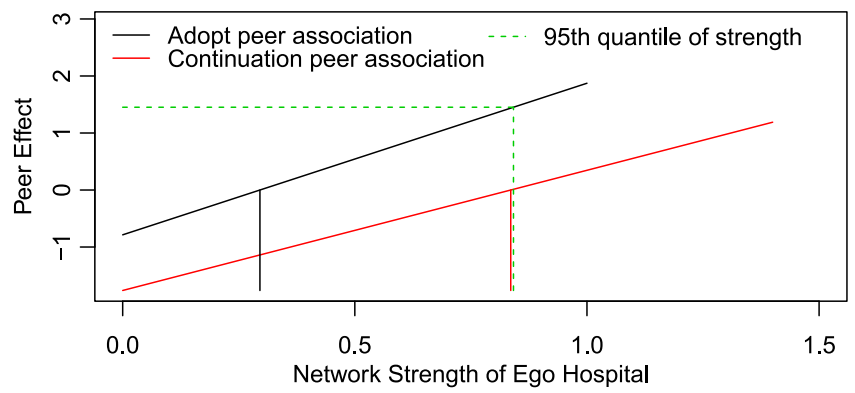

Referral and Implant Volume Model Peer Effects

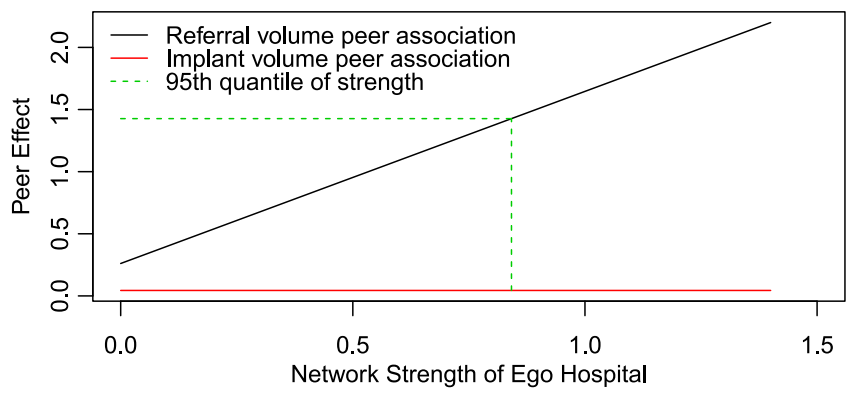

F I G U R E 3 Interaction effect plots of peer effect modification by the network strength of the ego hospital. The upper panel shows the log-odds of adopting ICD capability if currently not ICD capable (black font) and the log-odds of continuing to be ICD-capable if currently ICD capable (red font) by the network strength of the ego hospital. The lower panel shows the peer effects of referral and implant volume for ICD noncapable (black font) and ICD capable (red font) hospitals, respectively, as a function of the network strength of the ego hospital. The green dashed line shows the effect-sizes at the 95 'th quantile of strength while the black and red vertical lines in the upper panel mark the values of strength at which the effects are 0 (ie, the value of strength at which the peer effects transition from negative to positive). It is clear that substantial modification of the peer effect by strength occurs in three out of the four scenarios (all except the ICD implant volume peer-effect for ICD-capable hospitals) [Color figure can be viewed at wileyonlinelibrary.com] 
T A B L E 6 Estimated peer-effect models of number of ICDs for ICD noncapable (303 HRRs, 3299 hospitals, 11,981 observations) and capable (303 HRRs, 1305 hospitals, 4069 observations) hospitals

\begin{tabular}{|c|c|c|c|c|c|c|}
\hline \multirow[b]{2}{*}{ Term } & \multicolumn{3}{|c|}{ Referral } & \multicolumn{3}{|c|}{ Implant } \\
\hline & Estimate & Z-stat & $p$-value & Estimate & Z-stat & $p$-value \\
\hline (Intercept) & -5.986 & -49.63 & 0.000 & -5.228 & -38.97 & 0.000 \\
\hline Year 2008 & 0.000 & & & 0.000 & & \\
\hline Year 2009 & 0.370 & 16.79 & 0.000 & 0.274 & 20.61 & 0.000 \\
\hline Year 2010 & 0.262 & 12.73 & 0.000 & 0.202 & 16.85 & 0.000 \\
\hline Year 2011 & 0.087 & 3.93 & 0.000 & 0.028 & 2.19 & 0.029 \\
\hline Offset(Log \# patients) & 1.000 & & & 1.000 & & \\
\hline Lag \# physicians (100s) & 0.052 & 1.42 & 0.155 & 0.034 & 2.41 & 0.016 \\
\hline Lag strength $\left(10^{5}\right)$ & -0.531 & -4.77 & 0.000 & 0.079 & 2.16 & 0.031 \\
\hline Lag (physical) closeness & -0.120 & -4.41 & 0.000 & 0.002 & 0.07 & 0.944 \\
\hline Lag fragmentation & -0.122 & -1.33 & 0.184 & -0.072 & -0.79 & 0.430 \\
\hline Lag \# ICDs (10s) & 0.055 & 4.46 & 0.000 & 0.005 & 2.45 & 0.014 \\
\hline Lag peer capable & 0.257 & 4.27 & 0.000 & -0.130 & -3.47 & 0.001 \\
\hline Lag peer capable*strength & 0.518 & 3.78 & 0.000 & & & \\
\hline Lag peer referral (100s) & 0.264 & 3.13 & 0.002 & -0.033 & -0.53 & 0.593 \\
\hline Lag peer referral*strength & 1.385 & 5.00 & 0.000 & & & \\
\hline Lag peer implant (100s) & 0.362 & 8.50 & 0.000 & 0.044 & 1.39 & 0.164 \\
\hline Lag closeness capable & 0.189 & 2.61 & 0.009 & 0.185 & 4.02 & 0.000 \\
\hline Var(hospital) & $0.19 \pm 0.44$ & & & $0.19 \pm 0.44$ & & \\
\hline $\operatorname{Var}(\mathrm{HRR})$ & $0.067 \pm 0.26$ & & & $0.025 \pm 0.16$ & & \\
\hline
\end{tabular}

their estimated regression coefficients are smaller than the effect of the remaining predictor when one is excluded, which is consistent with the effect of one partially confounding the effect of the other. However, the correlation between the estimated regression coefficients of the lagged peer ICD capability and the lagged ICD capability of nearby hospitals in the estimated ICD adoption model in Table 5 is -0.294 , implying that the level of colinearity between these predictors is not of concern. Analogous comments apply to the subsequent models.

In the ICD continuation model, a positive regression coefficient implies higher values of that predictor are associated with increased likelihood of retention of ICD capability for ego hospitals that were ICD capable in the prior year. The estimated peer ICD capability-ego strength interaction is positive and highly significant (log-odds 2.108) as seen in Table 5 and its effect trajectory, $-1.762+2.108 \times$ strength. The overall effect of the network peer variables was significant (their addition decreased the deviance of the base model by $11.68, p=0.003$ ). The estimated peer-effect changes from negative to positive at the 94.9 th percentile of strength (equal to 0.836 ). Thus, although high peer ICD capability helps maintain the capability status of hospitals with high network strength, it encourages deadoption in less strongly connected hospitals. Such hospitals may find it too challenging to attract sufficiently many ICD patients to justify maintaining their ICD capability.

The estimated coefficients of the control predictors in both the adoption and deadoption models make intuitive sense. More attributed patients and physicians, being closer to other hospitals, higher fragmentation, and a greater number of patients receiving ICDs in the prior year are all associated with an increased likelihood of adopting ICD capability. In contrast, only being physically close to other hospitals and a greater number of attributed patients who received ICDs in the prior year are significantly associated with the likelihood of a capable hospital continuing as ICD capable. The estimated variance parameters reveal that there is substantially more unexplained variation between hospitals and regions (HRRs) in ICD adoption than ICD continuation (implying $\tau_{0}^{2}>\tau_{1}^{2}, \omega_{0}^{2}>\omega_{1}^{2}$ ) and that in general more variation resides between hospitals than between HRRs (implying $\tau_{q}^{2}>\omega_{q}^{2}, q=0,1$ ). 


\section{2 | Models of number of ICDs}

Network strength is significantly associated with ICD volume at noncapable hospitals through its modification of the effects of both the peer capable (log risk-ratio $0.518, p<0.001$ ) and the peer referral volume (log risk-ratio $1.385, p<0.001$ ) predictors (Table 6 and see the lower panel of Figure 3 for the referral volume peer effect). Furthermore, the main effects of each of the three peer ICD variables, including the implant volume association (log risk-ratio $0.362, p<0.001$ ), are highly statistically significant. The estimated regression coefficient of the proportion of physically close hospitals that are ICD capable of $0.189(p=0.009)$ is consistent with the notion that a greater supply of ICD capable hospitals induces more referrals from noncapable hospitals. The overall effect of the network peer variables was substantially greater than in any other model (their inclusion reduces the deviance by 156.1, $p<0.0001$ ).

In contrast to the ICD referral volume model, the network strength interaction variable coefficients in the ICD implant volume model were far from statistically significant, leading to the removal of all peer interaction variable predictors from this model. In general, the peer hospital predictors have smaller associations with the number of ICDs implanted at ICD capable hospitals than for the corresponding association in the noncapable ICD-volume model (Table 6 and as illustrated by the reduction in the deviance statistic of $15.3, p=0.0016)$. The lone exception is the peer ICD capable predictor whose significant negative association $(-0.130, p<0.001)$ implies that having strong network ties to other ICD capable peer hospitals is associated with performing fewer ICDs. However, the presence of a high proportion of nearby hospitals that are capable is associated with a ICD capable ego hospital performing more ICD implants per capita (log-risk-ratio 0.185, $p=0.001$ ), suggesting that local markets with many ICD capable hospitals may lead to greater volume of ICD procedures at each capable hospital in the market.

The estimated hospital variance components have similar magnitudes across the models, but the estimated HRR variance component is much greater in the referral volume than the implant volume model (implying $v_{0}^{2}>v_{1}^{2}$ ), suggesting that the ICD volume of noncapable hospitals is less predictable.

\section{CONCLUSION}

The key methodological contributions in this paper culminate in the development of a statistical model for simultaneously modeling multiple peer effects and their possible dependence on the ego hospital's structural importance in the network. This model advances the study of diffusion in health care by allowing peer effects acting on a hospital (or other organization) to be modified by the structure of that hospital's network strength. When structural importance is represented by strength, the overall peer effect is represented by a relative or strength-independent component and a total or aggregate component. If technologies diffuse through a restricted group of hospitals whose membership correlates with the strength of their network strength, the models developed herein are ideally suited to detecting such hospitals and the presence of hospital-to-hospital diffusion among them.

The incorporation of peer-effect modification by ego strength had a major impact on the results of the ICD peer effect analyses for the adoption and continuation of ICD capability. Peer effects are positive for the most strongly connected hospitals and negative for those less strongly connected, particularly with regard to continuation of ICD capability. Thus, for the peer effect of ICD adoption to be positive a hospital needs to be strongly connected in the network and for the same peer effect to increase the likelihood of a hospital remaining ICD capable that hospital needs to be among the most strongly connected hospitals. This combination of findings implies that ICD capable hospitals have over time become characterized by membership of a sub-network of the most strongly connected hospitals and that this trend will become even more enhanced in the future. We conjecture that the results for ICDs will be indicative of those for other medical technologies that are costly to setup and for whom sufficient time following market release has elapsed for the initial mass uptake (which often follows a logistic or "S-shaped" curve) to have been supplanted by competitive jockeying for market position and comparison-making to influential peers.

Because the data are observational the estimates obtained are associations as opposed to causal effects. By controlling for potential confounding variables such as the extent to which other hospitals are nearby and the ICD-status of nearby hospitals, we demonstrated that the observed peer effects are not merely a manifestation of market effects due to nearby hospitals and thus that knowledge of the network has the potential to explain important variation in the adoption of ICD capability and the level of ICD utilization. However, unmeasured confounders could have biased the results. For example, the number of US health systems that encompass multiple hospitals and physician practices has recently grown. It is reasonable to expect that peer-hospital influence might be different within a system than between a system. However, the 
direction of such a bias is not clear; a system may incentivize its hospitals to specialize due to need met elsewhere in the system, thus negating peer effects, or to adopt the strategies and follow the technology acquisitions of the most successful hospitals with support to do so provided by the system, inducing peer effects. Given data on health system membership, a natural analysis is to test whether presence in the same system modifies any of the results herein and so is a topic for future research. Another possible source of confounding is statewide certificate of need (CON) programs that require a hospital to prove a community need before being allowed to adopt a new technology. The presence of CON programs would likely push peer effects toward 0. Although CON programs still exist, not all states have them and many that did have mothballed them.

A causal interpretation of our results must also assume that the status and evolution of interhospital relationships (formation, dissolution, strengthening, weakening) do not depend on the same factors that affect ICD capability and utilization. ${ }^{47}$ If not, the phenomena popularly known as "birds of a feather flock together" or "homophily," which may arise from the seeking and sharing of patients between hospitals with similar characteristics, treatment tendencies, and clinical or other staff will present as endogenous selection. Distinguishing peer effects from homophily and unmeasured confounding is exceedingly difficult. ${ }^{48}$ One approach is to use instrumental variables but this relies on exact identifying assumptions that may be difficult to justify, especially for network data. ${ }^{49,50}$ Alternatively, one may jointly model the influence and selection processes. ${ }^{51,52}$ However, such models rely on untestable parametric assumptions, raising model robustness concerns. In lieu of these strategies, use of lagged peer variables partially mitigate the potential for bias from homophily and other forms of unmeasured confounding bias.

Another concern is that the network is obtained by projecting a bipartite patient-hospital network to a unipartite hospital-hospital network as opposed to directly observing formal relationships between hospitals. ${ }^{44}$ In a cross-sectional analysis this would raise the concern that a mechanical correlation arises from some of the patients used to construct the network also contributing to the peer and outcome variables for a hospital dyad (eg, a patient treated at many hospitals is more likely to receive an ICD than a patient who only visited a single hospital). The use of longitudinal models with lagged predictors also alleviates this concern as it separates observations used to construct the network from those analyzed as dependent variables.

An interesting area of future research concerns the specification of $\boldsymbol{W}_{t}$, for which methods may be extended in several ways beyond those considered in this paper. A first consideration is to account for directed networks, with one methodological extension being the incorporation of different weight matrices and thus peer effects for inward and outward networks ties with respect to the focal actor. Second, triadic dependence could be formerly incorporated. Although triadic and higher-order forms of dependence than dyadic dependence are typically spoken of in relation to models of the network itself, the concept of dyadic dependence could be brought to bear on the modeling of peer effects. The weight matrix $\boldsymbol{W}_{t}$ could be nonlinear in the sense that the weight reflecting the relative influence of one actor on another was increased in accordance to how many third actors the two have in common. As long as $\boldsymbol{W}_{t}$ is fully specified (ie, has a known value), the methods in this paper apply as $\boldsymbol{W}_{t}$ is conditioned on, not the subject of the model. A further extension would be to parameterize $\boldsymbol{W}_{t}$ with unknown parameters quantifying the extent and form of the nonlinearity either within the family of models considered in this paper or by adapting models of the network itself. ${ }^{53}$ These are topics for future research.

Despite the above challenges, estimating peer associations is a useful first step toward motivating deeper investigations to more rigorously seek to distinguish cause from association, including the use of randomized studies. Finding conclusive evidence of peer effects would imply that widespread improvements in healthcare organization can be achieved by intervening on a subset of hospitals judiciously selected based on their position in the network to maximize influence or spillover effects to other hospitals. ${ }^{54}$ The findings of this paper are a step toward this goal and to untapping the potential to improve medical practice through selective seeding of hospitals at which to apply interventions whose effects are designed to spillover to nonselected hospitals via peer effects.

\section{ACKNOWLEDGEMENTS}

The research in this paper was supported by NIH grants U01 AG046830 and P01 AG019783.

\section{CONFLICT OF INTEREST}

The authors declare no potential conflict of interest. 


\section{DATA AVAILABILITY STATEMENT}

The data used for the motivating analyses contain patient identifiable information and so cannot be made available. However, R code for performing the analyses and a simulated data set for illustrating its use may be obtained upon request from the corresponding author.

\section{ORCID}

\section{A. James O'Malley (1) https://orcid.org/0000-0002-5553-8874}

\section{REFERENCES}

1. Fisher ES, Wennberg DE, Stukel TA, Gottlieb DJ. Variations in the longitudinal efficiency of academic medical centers. Health Aff. 2004; Supplemental Web Exclusive; 23:19-32.

2. Fisher ES, Wennberg DE, Stukel TA, Gottlieb DJ, Lucas FL, Pinder EL. The implications of regional variations in Medicare spending. Part 1: the content, quality, and accessibility of care. Ann Intern Med. 2003;138:273-287.

3. Fisher ES, Wennberg DE, Stukel TA, Gottlieb DJ, Lucas FL, Pinder EL. The implications of regional variations in Medicare spending. Part 2: health outcomes and satisfaction with care. Ann Intern Med. 2003;138:273-287.

4. Wennberg JE. Tracking Medicine: A Researcher's Quest to Understand Health Care. New York, NY: Oxford University Press; 2010.

5. Newhouse JP, Garber AM, Graham RP, McCoy MA, Mancher M, Kibria A. Variation in Health Care Spending: Target Decision Making, Not Geography. Board on Health Care Services: Institute of Medicine; 2013.

6. TDI. The Dartmouth Atlas of Health Care. Lebanon, New Hampshire: The Dartmouth Institute for Health Policy and Clinical Practice. 2013. http://www.dartmouthatlas.org/.

7. Coleman J, Katz E, Menzel H. The diffusion of an innovation among physicians. Sociometry. 1957;20(4):253-270.

8. Waller LA, Gotway CA. Applied Spatial Statistics for Public Health Data. Hoboken, NJ: Wiley Interscience; 2004.

9. Banerjee S, Carlin BP, Gelfand AE. Hierarchical Modeling and Analysis for Spatial Data. Boca Raton, FL: Chapman and Hall; 2004.

10. Kapoor M, Kelejian HH, Prucha IR. Panel data models with spatially correlated error components. J Econ. 2007;140(1):97-130.

11. Kelejian HH, Prucha IR. HAC estimation in a spatial framework. J Econ. 2007;140(1):131-154.

12. Doreian P. Linear-models with spatially distributed data- spatial disturbances or spatial effects. Sociol Methods Res. 1980;9:29-60.

13. Dow MM. A biparametric approach to network autocorrelation. Sociol Methods Res. 1984;13:201-217.

14. Doreian P. Network autocorrelation models: problems and prospects. In: Griffith DA, ed. Spatial statistics: Past, present, future. Ann Arbor, MI: Michigan Document Services; 1989:369-389.

15. Friedkin NE. Social networks in structural equations models. Soc Psychol Q. 1990;53:316-328.

16. Leenders R. Modeling social influence through network autocorrelation: constructing the weight matrix. Soc Net. 2002;24:21-47.

17. Dittrich D, Leenders RTAJ, Mulder J. Bayesian estimation of the network autocorrelation model. Soc Netw. 2017;48:213-236.

18. Dittrich D, Leenders RTAJ, Mulder J. Network autocorrelation modeling: a Bayes factor approach for testing (multiple) precise interval hypotheses. Sociol Methods Res. 2017;48(3):642-676.

19. Christakis NA, Fowler JH. The spread of obesity in a large social network over 32 years. N Engl J Med. 2007;357:370-379.

20. Norton EC, Lindrooth RC, Ennett ST. Controlling for the endogeneity of peer substance use on adolescent alcohol and tobacco use. Health Econ. 1998;7(5):439-453.

21. Rosenquist JN, Fowler JH, Murabito J, Christakis NA. The spread of alcohol consumption behavior in a large social network. Ann Intern Med. 2010;152(7):426-433.

22. Keating N, Ayanian J, Cleary P, Marsden P. Factors affecting influential discussions among physicians: a social network analysis of a primary care practice. J Gen Intern Med. 2007;22(6):794-798.

23. Iwashyna TJ, Christie JD, Kahn JM, Asch DA. Uncharted paths: hospital networks in critical care. Chest. 2009;135:827-833.

24. Horwitz JR, Nichols A, Nallamothu BK, Sasson C, Iwashyna TJ. Expansion of invasive cardiac services in the United States. Circulation. 2013;128:803-810.

25. Pollack CE, Soulos PR, Gross CP. Physician's peer exposure and the adoption of a new cancer treatment modality. Cancer. 2015;121(16):2799-2807.

26. Pollack CE, Soulos PR, Herrin J, et al. The impact of social contagion on physician adoption of advanced imaging tests in breast cancer. J Natl Cancer Inst. 2017;109(8):djw330.

27. Landon BE, Keating NL, Barnett ML, et al. Variation in patient-sharing networks of physicians across the United States. J Am Med Assoc. 2008;308:265-273.

28. Lee BY, Song Y, Bartsch SM, Kim DS, Singh A, Avery TR. Long-term care facilities: important participants of the acute care facility social network? PLoS One. 2011;6(12):e29342.

29. Lomi A, Mascia D, Vu DQ, Pallotti F, Conaldi G, Iwashyna TJ. Quality of care and interhospital collaboration: a study of patient transfers in Italy. Med Care. 2014;52(5):407-414.

30. Mandl KD, Olson KL, Mines D, Liu C, Tian F. Provider collaboration: cohesion, constellations, and shared patients. J Gen Intern Med. 2014;29(11):1499-1505.

31. Pham HH, Schrag DS, O'Malley AS, Wu B, Bach PB. Care patterns in medicare and their implications for pay for performance. $N$ Engl J Med. 2007;356:1130-1139. 
32. Bynum JPW, Bernal-Delgado E, Gottlieb D, Fisher E. Assigning ambulatory patients and their physicians to hospitals: a method for obtaining population-based provider performance measurements. Health Serv Res. 2007;42:45-62.

33. Moen EL, Bynum JPW, Austin AM, Chakraborti G, Skinner JS, O'Malley AJ. Assessing variation in implantable cardioverter defibrillator therapy guideline adherence with physician and hospital patient-sharing networks. Med Care. 2017;56(4):350-357.

34. Lyons R. The spread of evidence-poor medicine via flawed social-network analysis. Stat, Politics, Policy. 2011;2(1):2.

35. O'Malley AJ, Marsden PV. The analysis of social networks. Health Serv Outcome Res Methodol. 2008;8(4):222-269.

36. Ord K. Estimation methods for models of spatial interaction. J Am Stat Assoc. 2012;70(349):120-126.

37. Anselin L. Spatial Econometrics: Methods and Models. Dordrecht, The Netherlands: Kluwer Academic Publishers; 1988.

38. Friedkin NEA. Structural Theory of Social Influence. Cambridge, UK: Cambridge University Press; 1998.

39. Anselin L. Spatial Econometrics. In: Badi B, ed. A Companion to Theoretical Econometrics. Oxford, UK: B. H. Blackwell Publishing Ltd; 2001.

40. Manski CA. Identification of endogenous social effects: the reflection problem. Rev Econ Stud. 1993;60:531-542.

41. Burt RS, Doreian P. Testing a structural model of perception: conformity and deviance with respect to journal norms in elite sociological methodology. Qual Quant. 1982;16:109-150.

42. O'Malley AJ. The analysis of social network data: an exciting frontier for statisticians. Stat Med. 2013;32:539-555.

43. O'Malley AJ, Onnela JP. Introduction to social network analysis: in health services evaluation section. In: Levy A, Goring S, Gatsonis C, Sobolev B, van Ginneken E, Busse R, eds. The Handbook of Health Services Research. New York, NY: Springer; 2019.

44. Fujimoto K, Chou CP, Valente TW. The network autocorrelation model using two-mode data: affiliation exposure and potential bias in the autocorrelation parameter. Soc Netw. 2011;33(3):231-243.

45. Bynum JPW, Andrews A, Sharp S, McCullough D, Wennberg JE. Fewer hospitalizations result when primary care is highly integrated into a continuing care retirement community. Health Aff. 2011;30(5):975-984.

46. Barnett ML, Landon BE, O'Malley AJ, Keating NL, Christakis NA. Mapping physician networks with self- reported and administrative data. Health Serv Res. 2011;46:1592-1609.

47. Noel H, Nyhan B. The "unfriending" problem: the consequences of homophily in friendship retention for causal estimates of social influence. Soc Netw. 2011;33:211-218.

48. Shalizi CR, Thomas AC. Homophily and contagion are generically confounded in observational social network studies. Sociol Methods Res. 2011;40(2):211-239.

49. O'Malley AJ, Elwert F, Rosenquist JN, Zaslavsky AM, Christakis NA. Estimating peer effects in longitudinal dyadic data using instrumental variables. Biometrics. 2010;70(3):506-515.

50. VanderWeele TJ, Ogburn EL, Tchetgen Tchetgen EJ. Why and when "flawed" social network analyses still yield valid tests of no contagion. Stat, Politics, Policy. 2012;3(1):Article number 4. https://doi.org/10.1515/2151-7509.1050.

51. Steglich CEG, Snijders TAB, Pearson M. Dynamic networks behavior: separating selection from influence. Sociol Methodol. 2010;40(1):329-393.

52. Leenders RTAJ. Longitudinal behavior of network structure and actor attributes: modeling interdependence of contagion and selection. In: Doreian P, Stokman F, eds. Evolution of Social Networks. New York: Gordon and Breach; 1997.

53. Robins GL, Pattison PE, Elliott P. Network models for social influence processes. Psychometrika. 2001;66:161-189.

54. Kim DA, Hwong AR, Stafford D, et al. A randomised controlled trial of social network targeting to maximise population behaviour change. Lancet. 2015;386(9989):145-153.

55. LeSage J, Pace RK. Introduction to Spatial Econometrics. New York: CRC Press; 2009.

How to cite this article: O'Malley AJ, Moen EL, Bynum JPW, Austin AM, Skinner JS. Modeling peer effect modification by network strength: The diffusion of implantable cardioverter defibrillators in the US hospital network. Statistics in Medicine. 2020;39:1125-1144. https://doi.org/10.1002/sim.8466

\section{APPENDIX: STEADY-STATE OF LONGITUDINAL MODEL}

We seek the steady-state distribution of Equation (3), the model specified in Section 3, but note that the following calculations can be adapted to other models (see page 26 of Le Sage and Pace $\left.{ }^{55}\right)$. Let $\epsilon_{t}=\left(\epsilon_{1 t}, \ldots, \epsilon_{n t}\right)^{T}$ so that $\operatorname{var}\left(\epsilon_{t}\right)=$ $\sigma^{2} \boldsymbol{I}$, where $\boldsymbol{I}$ is the $n \times n$ identity matrix. The model in Equation (3) in vectorized form is given by

$$
\boldsymbol{Y}_{t}=\beta_{1} \boldsymbol{Y}_{t-1}+\boldsymbol{X}_{t-1} \boldsymbol{\beta}_{2}+\alpha_{1} \boldsymbol{W}_{t-1} \boldsymbol{Y}_{t-1}+\epsilon_{t}
$$

Furthermore, suppose that there is minimal variation in $\boldsymbol{W}_{t}$ so that it may be approximated by a time invariant $\boldsymbol{W}$ and that $\boldsymbol{X}_{t} \rightarrow \boldsymbol{X}$. 
Theorem 1. If the principal eigenvalue of $\beta_{1} \boldsymbol{I}+\alpha_{1} \boldsymbol{W}$ is less than 1 and $\left(1-\beta_{1}\right) \boldsymbol{I}-\alpha_{1} \boldsymbol{W}$ is nonsingular then

$$
\lim _{t \rightarrow \infty} E\left[\boldsymbol{Y}_{t} \mid \boldsymbol{X}_{t-1}\right]=\left(\boldsymbol{I}-\beta_{1} \boldsymbol{I}-\alpha_{1} \boldsymbol{W}\right)^{-1} \boldsymbol{X} \boldsymbol{\beta}_{2}
$$

and

$$
\lim _{t \rightarrow \infty} \operatorname{var}\left(\boldsymbol{Y}_{t} \mid \boldsymbol{X}_{t-1}\right)=\sigma^{2}\left\{\boldsymbol{I}-\left(\beta_{1} \boldsymbol{I}+\alpha_{1} \boldsymbol{W}\right)\left(\beta_{1} \boldsymbol{I}+\alpha_{1} \boldsymbol{W}^{T}\right)\right\}^{-1} .
$$

Furthermore, the steady-state distribution for hospital i satisfies

$$
Y_{i}=\tilde{\alpha}_{1} W Y_{i}+\boldsymbol{X}_{i}^{T} \tilde{\boldsymbol{\beta}}_{\mathbf{1}}+\epsilon_{i}, i=1, \ldots, n,
$$

where $\tilde{\alpha}_{1}=\alpha_{1} /\left(1-\beta_{1}\right)$ and $\tilde{\beta}_{1}=\beta_{2} /\left(1-\beta_{1}\right)$.

Proof. Expressing the model in vector form it follows that

$$
\begin{aligned}
\boldsymbol{Y}_{t} & =\alpha_{1} \boldsymbol{W} \boldsymbol{Y}_{t-1}+\beta_{1} \boldsymbol{Y}_{t-1}+\boldsymbol{\beta}_{3}^{T} \boldsymbol{X}_{t-1}+\boldsymbol{\epsilon}_{t} \\
& =\left(\beta_{1} \boldsymbol{I}+\alpha_{1} \boldsymbol{W}\right)^{2} \boldsymbol{Y}_{t-2}+\left(\beta_{1} \boldsymbol{I}+\alpha_{1} \boldsymbol{W}\right)\left(\boldsymbol{X}_{t-2} \boldsymbol{\beta}_{2}+\boldsymbol{\epsilon}_{t-1}\right) \boldsymbol{\beta}_{2} \boldsymbol{X}_{t-1}+\boldsymbol{\epsilon}_{t} \\
& =\ldots\left(\beta_{1} \boldsymbol{I}+\alpha_{1} \boldsymbol{W}\right)^{t} \boldsymbol{Y}_{0}+\sum_{j=0}^{t-1}\left(\beta_{1} \boldsymbol{I}+\alpha_{1} \boldsymbol{W}\right)^{j}\left(\boldsymbol{X}_{t-j-1} \boldsymbol{\beta}_{2}+\boldsymbol{\epsilon}_{t-j}\right) .
\end{aligned}
$$

If the principal eigenvalue of $\beta_{1} I+\alpha_{1} \boldsymbol{W}$ is within the interval $(0,1)$, it follows that

$$
\sum_{j=0}^{(t-1)}\left(\beta_{1} \boldsymbol{I}+\alpha_{1} \boldsymbol{W}\right)^{j}=\left\{\boldsymbol{I}-\left(\beta_{1} \boldsymbol{I}+\alpha_{1} \boldsymbol{W}\right)^{t}\right\}\left(\boldsymbol{I}-\beta_{1} \boldsymbol{I}-\alpha_{1} \boldsymbol{W}\right)^{-1} .
$$

Therefore, as $t \rightarrow \infty$ the model converges to a system of equations satisfying

$$
\boldsymbol{Y}_{t}=\left(\boldsymbol{I}-\beta_{1} \boldsymbol{I}-\alpha_{1} \boldsymbol{W}\right)^{-1}\left(\boldsymbol{X}_{t-j-1} \boldsymbol{\beta}_{2}+\boldsymbol{\epsilon}_{t-j}\right)
$$

showing that, if it exists, the expectation at equilibrium is independent of the initial state, $\boldsymbol{Y}_{0}$. Hence, if $\boldsymbol{X}_{t} \rightarrow \boldsymbol{X}$ as $t \rightarrow \infty$

$$
\lim _{t \rightarrow \infty} E\left[\boldsymbol{Y}_{t} \mid \boldsymbol{X}_{t}\right]=\left(\boldsymbol{I}-\beta_{1} \boldsymbol{I}-\alpha_{1} \boldsymbol{W}\right)^{-1} \boldsymbol{X} \boldsymbol{\beta}_{2}
$$

and as $\operatorname{var}\left(\boldsymbol{\epsilon}_{t}\right)=\sigma^{2} \boldsymbol{I}$ it follows that

$$
\operatorname{limvar}_{t \rightarrow \infty}\left(\boldsymbol{Y}_{t} \mid \boldsymbol{X}_{t}\right)=\sigma^{2}\left(\boldsymbol{I}-\beta_{1} \boldsymbol{I}-\alpha_{1} \boldsymbol{W}\right)^{-1}\left(\boldsymbol{I}-\beta_{1} \boldsymbol{I}-\alpha_{1} \boldsymbol{W}\right)^{-T} .
$$

Because $\left(\beta_{1} \boldsymbol{I}+\alpha_{1} \boldsymbol{W}\right)\left(\beta_{1} \boldsymbol{I}+\alpha_{1} \boldsymbol{W}\right)^{T}$ has principal eigenvalue $\left(\beta_{1}+\alpha_{1}\right)^{2}$, if $\left|\beta_{1}+\alpha_{1}\right|<1$ then a steady-state distribution exists and Equation (A5) is the variance of $\boldsymbol{Y}_{t}$ when in steady state. Re-parameterizing and using results for multivariate normal distributions, it follows immediately that if $\left|\beta_{1}+\alpha_{1}\right|<1$ then the steady-state distribution is that of the cross-sectional model in Equation (A3).

The steady state results for Equation (3) do not easily generalize to Equation (8) as the involvement of $S_{i(t-1)} W Y_{i(t-1)}$ on the right-hand-side of the model inhibits closed-form derivations of the conditions under which the model converges to a steady state. However, the further $\alpha_{2}$ in Equation (8) is from 0, the less likely the model is to converge. 